\title{
Talker-specific learning in speech perception
}

\author{
LYNNE C. NYGAARD \\ Emory University, Atlanta, Georgia \\ and \\ DAVID B. PISONI \\ Indiana University, Bloomington, Indiana
}

\begin{abstract}
The effects of perceptual learning of talker identity on the recognition of spoken words and sentences were investigated in three experiments. In each experiment, listeners were trained to learn a set of 10 talkers' voices and were then given an intelligibility test to assess the influence of learning the voices on the processing of the linguistic content of speech. In the first experiment, listeners learned voices from isolated words and were then tested with novel isolated words mixed in noise. The results showed that listeners who were given words produced by familiar talkers at test showed better identification performance than did listeners who were given words produced by unfamiliar talkers. In the second experiment, listeners learned novel voices from sentence-length utterances and were then presented with isolated words. The results showed that learning a talker's voice from sentences did not generalize well to identification of novel isolated words. In the third experiment, listeners learned voices from sentence-length utterances and were then given sentence-length utterances produced by familiar and unfamiliar talkers at test. We found that perceptual learning of novel voices from sentence-length utterances improved speech intelligibility for words in sentences. Generalization and transfer from voice learning to linguistic processing was found to be sensitive to the talker-specific information available during learning and test. These findings demonstrate that increased sensitivity to talker-specific information affects the perception of the linguistic properties of speech in isolated words and sentences.
\end{abstract}

During everyday conversation, listeners effortlessly understand talkers with a wide variety of individual vocal characteristics and styles. It is only when we encounter an unfamiliar talker with an unusual dialect or accent that we become consciously aware that we have to adjust to the idiosyncratic vocal attributes of a novel talker. Presumably, this adjustment involves a period of perceptual adaptation in which listeners learn to differentiate the unique properties of each talker's speech patterns from the underlying intended linguistic message. Listening to speech produced by talkers of different dialects and accents is an extreme example of what occurs routinely as we encounter unfamiliar talkers. The purpose of the present investigation was to study the process of perceptual learning and adaptation to individual talkers and to determine how sensitivity to talker identity affects the intelligibility of the linguistic aspects of speech - specifically, to study the recognition of spoken words in isolation and in sentence contexts.

This research was supported by NIDCD Research Grant DC-00111 and NIDCD Research Training Grant DC-00012 to Indiana University. Portions of this research were presented at the 125th meeting of the Acoustical Society of America in Ottawa and at the XIIIth International Congress of Phonetic Sciences in Stockholm. The authors would like to thank Luis Hernandez for his technical expertise, Matt Peuquet for his work on stimulus materials and experiment running, and Mike Kalish for his help with multidimensional scaling. Correspondence concerning this article should be addressed to L. C. Nygaard, Department of Psychology, 532 N. Kilgo Circle, Emory University, Atlanta, GA 30322 (e-mail: nygaard@social-sci.ss.emory.edu).

\section{The Abstractionist Approach to Speech Perception}

Traditionally, the perception of the linguistic content of speech - the words, phrases, and sentences of an utterance-has been studied separately from the perception of talker identity (Pisoni, 1997). Research on the perception of the linguistic aspects of spoken language has considered variation in the acoustic realization of linguistic components due to differences in individual talkers as a source of noise that serves to obscure the underlying abstract symbolic linguistic message. Variability is considered a perceptual problem that listeners must solve if they are to recover the linguistic constituents that carry meaning (Shankweiler, Strange, \& Verbrugge, 1977). The proposed solution to this problem of talker variability is that there is a perceptual normalization process in which talker-specific acoustic-phonetic properties are evaluated in relation to prototypical mental representations (Joos, 1948; Ladefoged \& Broadbent, 1957; Pisoni, 1997; Summerfield \& Haggard, 1973). Variation is assumed to be stripped away so that the listener can arrive at canonical representations that underlie further linguistic analysis. Implicit in this view of perceptual normalization is the assumption that the end product of perception is a series of abstract, symbolic, idealized, linguistic units (Halle, 1985; Joos, 1948; Kuhl; 1991, 1992; Pisoni, 1997).

This abstractionist approach to the perception of spoken language with its emphasis on context-free processing units falls short of providing a satisfactory explanation for the relationship between the processing of linguistic 
content and the analysis of a talker's voice. Although the speech signal carries a considerable amount of "personal" information about the talker along with the linguistic content into the communicative setting (Ladefoged \& Broadbent, 1957; Laver, 1989; Laver \& Trudgill, 1979; Van Lancker, 1991), little, if any, role for talker information has been assumed in current theoretical accounts of the perception of speech or spoken language processing. A separate body of research has addressed the perception and identification of talker identity, viewing the speech signal as simply a carrier of talker information (e.g., Legge, Grossmann, \& Pieper, 1984; Van Lancker, Kreiman, \& Emmorey, 1985). This explicit dissociation of research involving linguistic processing, on the one hand, and voice perception, on the other hand, reflects an implicit theoretical separation. Talker identification and perception are assumed to involve a distinct set of perceptual mechanisms which operate on attributes of the acoustic speech signal that are separate and autonomous from the attributes that underlie spoken word recognition and comprehension of the linguistic message (Van Lancker, 1991; Van Lancker, Cummings, Kreiman, \& Dobkin, 1988; Van Lancker \& Kreiman, 1987; Van Lancker, Kreiman, \& Emmorey, 1985).

An alternative to the abstractionist approach to speech perception and spoken language recognition suggests that the traditional view of perceptual normalization and its long-standing emphasis on the search for abstract, canonical linguistic units as the endpoint of perception may need to be reconsidered or abandoned entirely ( $\mathrm{Pi}$ soni, 1997). Over the last few years, a number of researchers have demonstrated that stimulus variability is a rich source of information that is encoded and stored in memory along with the linguistic content of a talker's utterance (e.g., Palmeri, Goldinger, \& Pisoni, 1993; Pisoni, 1993). These findings suggest that speech perception does not involve a mapping of invariant attributes or features in the signal onto idealized symbolic representations in memory, but rather employs highly detailed and specific encodings of speech which preserve many attributes of the acoustic signal (Goldinger, 1992, 1996).

\section{The Role of Indexical Information}

The human voice conveys a considerable amount of information about a speaker's physical, social, and psychological characteristics, and these aspects of speech, referred to as indexical information (Abercrombie, 1967), complement the processing of linguistic content during spoken communication. Individuals differ in the size and shape of their vocal tracts (Fant, 1973; Joos, 1948; Peterson \& Barney, 1952) and in their idiosyncratic methods of articulation (Ladefoged, 1980), as well as in their individual glottal characteristics. These properties provide information atout a speaker's identity (Van Lancker, Kreiman, \& Emmorey, 1985; Van Lancker, Kreiman, \& Wickens, 1985) in addition to more general information about a speaker's origin and background (Labov, 1972). The speech signal also provides important information about more short-term aspects of a speaker's voice, such as physical, emotional, or psychological states. These psychological factors are readily perceived when anger, depression, or happiness is recognized in a speaker's voice (Costanzo, Markel, \& Costanzo, 1989; Markel, Bein, \& Phillis, 1973; Murray \& Arnott, 1993).

In everyday conversation, the indexical properties of the speech signal become quite important as perceivers use this information to govern their own speaking styles and responses. From more permanent characteristics of a speaker's voice that provide information about identity to the short-term vocal changes related to emotion or "tone of voice," indexical information contributes to the overall interpretation of a speaker's utterance. How, then, is the perception and encoding of the indexical properties of the speech signal related to the analysis of the more abstract linguistic content of an utterance? The essence of the problem is that both types of information are conveyed simultaneously along the same acoustic dimensions within the speech signal (Remez, Fellowes, \& Rubin, 1997). As the acoustic wave form of a talker's utterance reaches the listener's ear, information about the talker must be disentangled from information about the linguistic content of the utterance. Consequently, any explanation of "perceptual normalization" for talker variability will necessarily need to include an account of the processing and representation of both the linguistic and the indexical information that are carried in parallel in the speech signal.

A number of recent experiments have been reported that explicitly address the relationship between linguistic analysis and talker variability. Several studies have shown that talker variability has a significant impact both on the perceptual processing of spoken utterances and on the memory representations constructed during the perception of spoken language. For example, talker variability has been shown to affect both vowel perception (Assmann, Nearey, \& Hogan, 1982; Summerfield, 1975; Summerfield \& Haggard, 1973; Verbrugge, Strange, Shankweiler, \& Edman, 1976; Weenink, 1986) and spoken word recognition (Cole, Coltheart, \& Allard, 1974; Creelman, 1957; Mullennix, Pisoni, \& Martin, 1989). Mullennix et al. (1989) found that perceptual identification of words presented in noise was significantly poorer when the words were produced by multiple talkers than when they were produced by a single talker (see also Somm.rs, Nygaard, \& Pisoni, 1994). In addition, using a Garner (1974) speeded classification task, Mullennix and Pisoni (1990) found that listeners had difficulty ignoring irrelevant variation in a talker's voice when asked to classify syllables by initial phoneme. When asked to classify the same stimuli according to the sex of the speaker, these listeners also had difficulty ignoring irrelevant variation in the initial phoneme. Taken together, these results suggest that variability due to changes in a talker's voice affects the recovery of the linguistic aspects of the speech signal. Aspects of the speech signal related to classifying talker identity seem to be integrally linked to attributes 
related to the processing of the linguistic content of the signal.

In a recent study, Remez et al. (1997) found that information encoded in sine wave replicas of spoken utterances also supports talker identification. These nonspeech signals are assumed to preserve only the time-varying phonetic information essential for linguistic interpretation and none of the acoustic attributes traditionally proposed to underlie the identification of the talker's voice (Bricker \& Pruzansky, 1976). Remez et al. found that listeners were able to discriminate and match to sample a set of sine wave replicas of utterances produced by unfamiliar talkers as well as identify sine wave replicas of utterances produced by a set of familiar talkers. These results show that timevarying phonetic information preserves some of the unique acoustic information that characterizes individual talkers' voices.

In addition to evidence linking talker variability to linguistic analysis in perception, there is now considerable evidence that talker information affects memory processes as well. Martin, Mullennix, Pisoni, and Summers (1989) found that serial recall of spoken word lists produced by multiple talkers was poorer than recall of lists produced by a single talker; but the result was found only in the primacy portion of the serial recall curve. Martin et al. interpreted these findings to suggest that variation in a talker's voice from word to word in a list competes for processing resources in the recall task. Analysis of talker information during a memory task appears to be both time- and resource-demanding, leaving fewer resources for the rehearsal and transfer of words into long-term memory. In addition, Martin et al. found that recall of a series of visually presented digits was poorer when followed by a multiple-talker list than when followed by a single-talker list, again suggesting that talker variability increases the capacity demands of the working memory system.

In a subsequent series of experiments, Goldinger, Pisoni, and Logan (1991) investigated the serial recall of multiple-talker and single-talker lists, using presentation rate as an additional experimental variable. Goldinger et al. found that at relatively fast presentation rates, serial recall in initial list positions was poorer for multipletalker lists than for single-talker lists, replicating the earlier findings of Martin et al. (1989). At slower presentation rates, however, recall performance was poorer in initial list positions for the single-talker rather than for the multiple-talker lists (see also Nygaard, Sommers, \& Pisoni, 1995). This interaction between presentation rate and serial recall for the multiple- and single-talker word lists suggests that at fast presentation rates, when processing is constrained by time, talker variability affects both the perceptual encoding and the rehearsal of items in the serial recall task. At slower presentation rates, when listeners have more time and resources to encode and rehearse talker information, they are able to use that information to aid them in the encoding of item and order information. These memory findings suggest that talker information may not be discarded in the process of spoken word recognition but rather is retained in memory along with the more abstract, symbolic linguistic content of the utterance.

A stronger demonstration that detailed talker-specific information is retained in long-term memory comes from another series of memory experiments conducted by Palmeri et al. (1993). Using a continuous recognition memory task (Shepard \& Teghtsoonian, 1961), Palmeri et al. found that talker-specific information is retained in memory along with lexical information, and that this information can facilitate listeners' recognition memory. In the continuous recognition memory task, listeners were asked to listen to a list of spoken words and identify each word as "old" or "new." Words repeated in the same voice were recognized better than words repeated in a different voice. This advantage for same-voice repetitions suggests that listeners are simultaneously processing attributes of the linguistic content and attributes of the talker's voice and that both sets of stimulus attributes are encoded and preserved in memory. Thus, variations in a talker's voice appear to be incorporated in memory into a highly detailed, rich representation of a talker's utterance (see also Craik \& Kirsner, 1974; Geiselman, 1979; Geiselman \& Bellezza, 1976, 1977; Geiselman \& Crawley, 1983; Goldinger, 1996).

Church and Schacter (1994) have reported similar findings in a series of experiments aimed at assessing implicit savings for surface characteristics of spoken language. Using an implicit memory paradigm to study priming, Church and Schacter found that repetition of surface characteristics such as a talker's voice, affective tone (happy or sad), and fundamental frequency from study to test phase of their task resulted in better implicit word identification than when prime and target were dissimilar from study to test along each of these dimensions. Explicit recognition memory was not affected by these manipulations. The explanation hypothesized for this implicit savings was that a general-purpose perceptual representation system operates in a modality-specific manner to preserve detailed instance-specific perceptual information (Schacter, 1990). This detailed perceptual information can then be used, in this case in addition to lexical content, to implicitly access prior events. These findings, taken together with those of Palmeri et al. (1993; see also Pollack, Pickett, \& Sumby, 1954), suggest that the effects of talker variability on perception and memory are a consequence of the additional processing time and resources that are devoted to encoding talker-specific information when the talker's voice changes from item to item in these tasks.

The research reviewed above makes a convincing case for the notion that talker-specific information is retained in memory and can be used as a cue, in addition to linguistic content, to retrieve specific linguistic events from memory. The question still remains, however, as to the relationship between the processing of talker information and the processing of linguistic content. Are perception of talker 
idertity and perception of linguistic content independent processes such that each contributes information separately about a to-be-remembered event? Or, are the perceptual analyses that extract both types of information integrally linked? In the present series of experiments, we sought to address these questions by focusing on the perceptual learning of novel voices.

\section{Perceptual Learning of Voices}

Relatively few studies have been conducted on the role of perceptual learning in the perception of speech and language in adults (but see Lively, Logan, \& Pisoni, 1993; Lively, Pisoni, Yamada, Tohkura, \& Yamada, 1994; Logan, Lively, \& Pisoni, 1991; Strange \& Dittmann, 1984). Although the role of categorization in perceptual sensitivity has long intrigued psychologists (E. J. Gibson, 1969, 1991; Goldstone, 1994; Wohlwill, 1958), increased perceptual sensitivity to aspects of the speech signal has traditionally been considered an interesting empirical demonstration rather than a routine aspect of our everyday perceptual experience. Yet, in our use of language, we are often aware that through exposure to and learning of a novel talker's voice, for example, we become increasingly able to recover the linguistic aspects of an utterance that seemed difficult to understand only moments earlier. The present investigation was designed to explicitly examine this role of the perceptual learning of a talker's voice in spoken language processing.

In a more general sense, it is possible to use the relationship between the learning of talker identity and linguistic processing as a test case for the study of perceptual learning in a highly complex natural stimulus domain such as speech. According to E. J. Gibson (1969), perceptual learning involves "an increase in the ability to extract information from the environment, as a result of experience and practice with stimulation coming from it" (p. 3). Gibson identified two types of perceptual learning. The first type suggests that perceptual sensitivity can be enhanced by preexposure to a set of stimuli, or "predifferentiation" (Hall, 1991). Mere experience of the stimulus domain increases perceivers' sensitivity. In the second type, explicit experience in categorizing or identifying stimuli allows perceivers to become attuned to specific diagnostic physical features (J. J. Gibson \& E. J. Gibson, 1955). For this type of learning, the organization of stimuli into categories has been shown to have an important influence on subsequent perceptual sensitivity (Goldstone, 1994). Within this domain of perceptual learning, Lawrence (1949) developed a theory of acquired distinctiveness of cues, such that cues or features that are relevant to a task become generally distinctive. In the case of talker learning, categorizing or identifying talker's voices may lead to increased distinctiveness of the perceptual dimensions of talker identity. If a benefit of perceptual learning of voice can be demonstrated for linguistic processing as well, it would suggest that the same underlying dimensions subserve both perceptual abilities.
Clues to the issues just raised come from experiments on talker identification and discrimination and from a handful of studies of perceptual learning of category structure in spoken language. Earlier research has shown that listeners can learn to identify a set of talkers from their voices alone (e.g., Doddington, 1985; Williams, 1964) and are quite good at discriminating among unfamiliar talkers (e.g., Van Lancker \& Kreiman, 1987). In these studies, it has been found that a number of factors, such as the a priori distinctiveness of the set of voices to be learned, the number of talkers to be identified or discriminated, and the length or duration of the utterances used during training (i.e., syllables, words, phrases, passages), can mediate learning of voices. Not surprisingly, listeners learn to recognize talkers' voices most readily when utterances of long duration from a few highly distinct talkers a:e used. These results suggest that a period of perceptual learning is required for listeners to become sensitive to talker-specific information in the speech signal. Listeners do not appear to acquire expertise in talker recognition effortlessly, but rather learn over time to attend explicitly to the unique, acoustically distinct properties of each talker's voice.

The crucial research question then becomes whether, given experience with the particular aspects of the speech signal relating to talker identity, it follows that listeners also become sensitive to talker-specific linguistic properties. Outside the domain of adaptation to voice, selective training on particular acoustic dimensions has been shown to modify even highly stable low-level phonetic categories. For example, Logan et al. (1991; see also Lively et al., 1993; Lively et al., 1994) have demonstrated perceptual learning of the $/ \mathrm{r} /-/ 1 /$ contrast by adult native speakers of Japanese. This contrast is not phonemic for native speakers of Japanese, and adult speakers have difficulty reliably categorizing instances from these categories. However, Logan et al. (as well as Lively et al., 1993; Lively et al., 1994) found, using a high-variability training program with explicit feedback, that native Japanese speakers can learn to discriminate the relevant acoustic dimensions and reliably classify $/ r /$ and $/ 1 /$. The authors argue that perceptual learning of nonnative contrasts is possible and suggest that a certain amount of neural plasticity exists in adult speech perception. Thus, perceptual mechanisms that subserve phonetic categorization in adults are susceptible to general processes of learning and adaptation.

In addition to perceptual learning of phonetic category . structure, perceptual adaptation to continuous synthesized speech has also been demonstrated in several studies. Greenspan, Nusbaum, and Pisoni (1988) showed that repeated practice in transcribing synthetic speech resulted in better comprehension performance. Exposure to the unique properties of synthetic speech resulted in better comprehension for novel instances of speech synthesized in the same manner. The learning, however, was specific to the training and testing materials used (see 
also Schwab, Nusbaum, \& Pisoni, 1985). Practice with synthesized sentences improved transcription performance for synthesized sentences and isolated words. Practice with isolated synthetic words improved word but not sentence transcription, suggesting that exposure during learning must be specific to the stimulus dimensions that will be relevant at test. Similarly, Dupoux and Green (1997) have found evidence for rapid perceptual adaptation to compressed speech. A group of listeners who received exposure to digitally compressed speech showed better subsequent transcription performance for compressed speech than did a group of listeners who were not previously exposed. Taken together, these practice effects with synthetic and compressed speech suggest that the speech processing system is capable of adjusting to a variety of distortions, both synthetic and natural, that occur in the acoustic signal. Furthermore, listeners do not appear to just become familiar with the sound of synthetic or compressed speech in these experiments, but rather show evidence of learning the specific acoustic-phonetic mapping rules that describe the relationship between the rulegoverned synthetic manipulations and each listener's underlying linguistic knowledge (see Greenspan et al., 1988).

The variation in spoken language that is introduced by individual talkers' speaking styles and vocal tract anatomies is analogous to the distortions imposed when speech is synthesized by rule. Each talker's vocal style shapes the acoustic realization of linguistic constituents in different but systematic and predictable ways. Nevertheless, perceptual adaptation to individual talkers' voices, as mentioned previously, has traditionally been cast as a problem of eliminating variation due to individual differences in speakers' voices from underlying linguistic constants, rather than as a perceptual learning process in which listeners become attuned to properties of the speech signal which subserve both talker identification and linguistic processing (Garvin \& Ladefoged, 1963; Johnson, 1990; Ladefoged \& Broadbent, 1957; Miller, 1989; Nearey, 1989). In this sense, perceptual adaptation is assumed to be a mandatory process in speech perception that works very quickly and automatically to strip away talkerspecific information. According to this view, perceptual learning of talker identity should be distinct from linguistic analysis (Ladefoged \& Broadbent, 1957; Miller, 1989; Nearey, 1989).

\section{The Present Experiments}

Demonstrating an influence of perceptual learning of novel voices on the recovery of the linguistic content of an utterance would provide important evidence for interdependence of the mechanisms subserving each function. The goal of the present investigation was to determine whether such a link exists and to assess the role of general cognitive processes such as perceptual learning, attention, and memory in the perception of spoken language.

In the first experiment, we sought to initially establish the effects of learning novel voices on the perception of isolated words. Listeners learned to identify a set of 10 voices ( 5 male and 5 female) over a 9-day period and were then asked to recognize novel words produced by talkers they either had or had not heard during training. The purpose of the experiment was twofold. First, we wanted to investigate the perceptual learning of voices in its own right. Would listeners be able to learn to identify talkers' voices from lists of short isolated words? Of interest were issues concerning the identifiability or distinctiveness of individual talkers as well as individual differences in listeners' abilities to learn the set of talkers. Second, given that listeners could successfully learn to identify a set of talkers, we sought to assess the effects of voice learning on their ability to recognize words mixed in noise. If words produced by familiar talkers are more easily recognized or more intelligible than words produced by unfamiliar talkers, this result would suggest that the perceptual learning in the talker identification task transferred to the word recognition task. This transfer of learning has several theoretical implications. One is that the perceptual learning of talker identity draws attention to the same perceptual attributes of the acoustic speech signal that are also important for word recognition. Therefore, the underlying representational code must somehow be integrated or linked in processing. Another implication is that mutual dependence of the perception of talker identity and linguistic identity would argue against traditional accounts of spoken language processing emphasizing abstract, context-free linguistic units. Instead, highly detailed information about the entire speech event is retained in long-term memory along with the more abstract linguistic content.

In two additional experiments, the processes of perceptual learning and generalization were explored in greater detail. Again, listeners were asked to learn a set of 10 novel voices. Then they attempted to identify the linguistic content of speech produced by familiar or unfamiliar talkers. However, in both of these experiments, the listeners learned to identify talkers from sentence-length utterances rather than from isolated words as in the first experiment. In one experiment, the listeners were asked to transcribe isolated words mixed in noise produced both by talkers learned during training and by a set of unfamiliar talkers. In the other experiment, the listeners were asked to transcribe sentence-length utterances mixed in noise produced by familiar and unfamiliar talkers. Our goal was to assess what type of talker-specific information is learned when listeners are trained with sentencelength versus word-length utterances and whether this learning would be task specific, generalizing only to similar test stimuli (Greenspan et al., 1988). We hypothesized that learning voices from isolated words would be a difficult task, focusing listeners' attention on detailed talker-specific acoustic-phonetic variation. Thus, we reasoned that any benefit or transfer from the dimension of talker identity to spoken words would be greatest when fine acoustic-phonetic distinctions were required, as in the isolated word recognition task. Learning voices from sentence-length utterances conversely was hypoth- 
esized to be a much easier learning task, focusing listeners' attention on more coarse grained, global attributes of talker identity such as prosody, intonation, and rhythm. We expected that listeners would show considerable transfer of learning to a sentence transcription task. Thus, in these experiments, it was assumed that listeners' attention would be drawn to different inventories of talker-specific information for sentence versus word materials. If so, the role of attention in perceptual learning could be assessed to determine the ultimate pattern of task-specific generalization and its benefit in spoken word recognition.

\section{EXPERIMENT 1}

In a recent study, Nygaard, Sommers, and Pisoni (1994) found that learning a talker's voice facilitated subsequent phonetic anaiyses. In their study, listeners were trained over a 9-day period to identify a set of five female and five male voices from isolated words and were then given a speech intelligibility task. During training, listeners were required to associate 1 of 10 common names with each voice and were given explicit feedback regarding their performance. The results showed that listeners who heard familiar talkers at test were better able to extract the linguistic content of isolated words than those who heard unfamiliar talkers at test. Their initial findings suggested that the perceptual learning of a talker's voice could modify the linguistic processing of isolated words.

The present investigation was designed te replicate and extend Nygaard et al.'s (1994) analyses of instancespecific factors in perceptual learning and to report additional data from this initial experiment. In Nygaard et al. (1994), large individual differences in listeners' abilities to learn the set of talkers were observed. Some listeners improved dramatically over the 9-day training period, while others showed little if any improvement. Nygaard et al. (1994) reported and analyzed data only from listeners who were able to learn the set of talkers' voices and who reached a minimum performance criterion. Of interest as well is the performance of listeners who were not able to learn the talkers' voices to criterion. These subjects provide an ideal comparison group, because although they received the same training and exposure to the set of talkers' voices, they were not able to attend specifically or successfully to the unique talker-specific aspects of the speech signal. Thus, in the present report, we assessed the nature of perceptual learning in these experiments by comparing listeners who learned the voices with listeners who did not learn the set of voices well enough to identify them reliably to criterion. The question of interest was whether the perceptual learning of voices that benefited spoken word recognition was due to the mere exposure of listeners to the set of talkers used or whether successful identification and categorization of voices would prove to be a necessary prerequisite to increase perceptual sensitivity to the linguistic content of familiar talkers' utterances (E. J. Gibson, 1969).
In addition to evaluating the nature of perceptual learning and transfer of training in this paradigm, we report detailed analyses of voice learning for all subjects. In these analyses, we address issues concerning individual differences in listeners' abilities (listener-specific factors) and differences in talker identifiability and intelligibility (talker-specific variables). Do individual listeners differ in their talker-learning strategies? Are all talkers equally identifiable? Are male and female voices different in terms of how easily they are learned? Our aim was to answer these questions by investigating in greater depth the factors that mediate voice learning and how perceptual sensitivity to voice is acquired under these learning conditions.

\section{Method}

\section{Listeners}

Sixty-six undergraduate and graduate students at Indiana University participated as listeners in this study. They were assigned to one of four conditions. Nineteen served in the trained experimental condition and 19 in the trained control condition. Fourteen served in each of the untrained control conditions. All were native speakers of American English and reported no history of a speech or hearing disorder at the time of testing. The listeners were paid for their participation.

\section{Stimulus Materials}

Three sets of stimuli were used in this experiment. All items were selected from a database of 360 monosyllabic words produced by 10 male and 10 female speakers taken from the vocabulary of the Modified Rhyme Test (House, Williams, Hecker, \& Kryter, 1965) and from phonetically balanced (PB) word lists (Egan, 1948). The talkers were all native speakers of American English between 20 and 50 years of age. There was no attempt to match any of the speakers for age or dialect. Each word was recorded on audiotape with the use of a high-quality professional microphone and was digitized at $10 \mathrm{kHz}$ with a 12-bit analog-to-digital converter. The root mean squared (RMS) amplitude levels for all words were digitally equated. Word identification tests in quiet showed greater than $90 \%$ intelligibility for all words. In addition, all words were rated to be highly familiar on a 7-point rating scale (Nusbaum, Pisoni, \& Davis, 1984).

\section{Procedure}

Training. The two groups of 19 listeners completed nine training sessions over a period of 2 weeks. They were asked to learn to recognize each talker's voice and to associate each voice with 1 of 10 common names (see Lightfoot, 1989). The digitized stimuli were presented with the use of a 12-bit digital-to-analog converter and were low-pass filtered at $4.8 \mathrm{kHz}$. The stimuli were presented to the listeners over matched and calibrated TDH-39 headphones at approximately $80 \mathrm{~dB}$ SPL.

During each of the nine hour-long training sessions, both groups of trained listeners completed three difference phases designed to acquaint them with the 10 different voices to be learned. The first phase was a familiarization task, in which 5 words from each of the 10 talkers were presented in succession to the listeners. Then, a 10-word list composed of 1 word from each talker was presented. As each item was presented to the listener, the name of the corresponding talker was displayed on a computer screen. The experimenter instructed the subjects to listen carefully to each word presented and to attempt to learn the name associated with each talker's voice. This familiarization procedure was intended to give the listeners some direct experience with the range of variability within cach talker's voice. 
The second phase of training consisted of a recognition task, in which 10 words from each of the 10 talkers were presented in random order to the listeners. The hundred words used in this phase did not overlap with those used in the first phase. The listeners were asked to identify the name of the talker who had produced each token, and they were given immediate feedback about the correct name after each trial. The listeners responded by pressing the appropriate key on a keyboard. Keys 1-5 were labeled with common male names (Bill, Joe, Mike, etc.), and Keys 6-10 were labeled with common female names (Sue, Mary, Carol, etc.).

During each training session, the listeners completed two repetitions of the first two phases of training and were then administered a test phase. As in the second phase of training, 10 words from each of the 10 talkers were mixed and presented in random order. The listeners were asked to identify each speaker's voice by choosing the appropriate name on each trial. To measure learning, feedback was not given during the test phase.

The same 100 words were used as stimuli for each of the training phases. However, the listeners never heard the same item produced by the same talker in both the test and the training phases on a given day. Furthermore, the stimuli were reselected from the database on each day of training, so that the listeners never heard the same item produced by the same talker in training. So, for example, the 10 words that were produced by one talker on the lst day of training would be produced by another talker on the 2 nd day of training. This procedure was intended to maximize the number of different tokens that listeners heard from each talker.

Generalization. During the 10th session of the experiment, both groups of listeners completed a generalization test that was identical to the test phase used during training except that a set of novel words produced by the same 10 talkers was used.

Transfer word intelligibility. In addition to the generalization test, the listeners were given a speech intelligibility test in which they were asked to identify isolated words presented in noise. One hundred novel words were presented at $80,75,70$, or $65 \mathrm{~dB}$ (SPL) mixed in continuous white noise that was low-pass filtered at $4.8 \mathrm{kHz}$ and presented at $70 \mathrm{~dB}$ (SPL). This procedure resulted in four signal-to-noise ratios: $+10,+5,0$, and $-5 \mathrm{~dB}$. Twenty-five words were presented at each signal-to-noise ratio. In this task, the listeners were asked to transcribe each word (rather than to identify the talker's voice) on each trial. The listeners in the rrained experimental group were presented with 10 words produced by each of the 10 talkers whom they had previously learned to identify during training. Listeners in the trained control group were presented with the same words produced by 10 new talkers ( 5 male and 5 female) whom they had not heard during training. In both cases, words from each talker were mixed and presented in random order.

In addition to giving the trained listeners (experimental and control) the word intelligibility test, two additional groups of 14 listeners were run to control for possible inherent intelligibility differences between the two sets of talkers' voices. These two control groups of listeners received only the word intelligibility tests and did not receive any training on voices prior to test. Thus, one group of untrained controls received the word intelligibility test that was given to the trained experimental group, and the other group of untrained controls received the word intelligibility test that was given to the trained control group.

Results
Training
The data from the two trained groups revealed large
individual differences in the listeners' voice identification
performance. Figure 1 shows scatterplots of these indi-
vidual listeners' performances from Day 9 of training,
plotted against their performances on the generalization
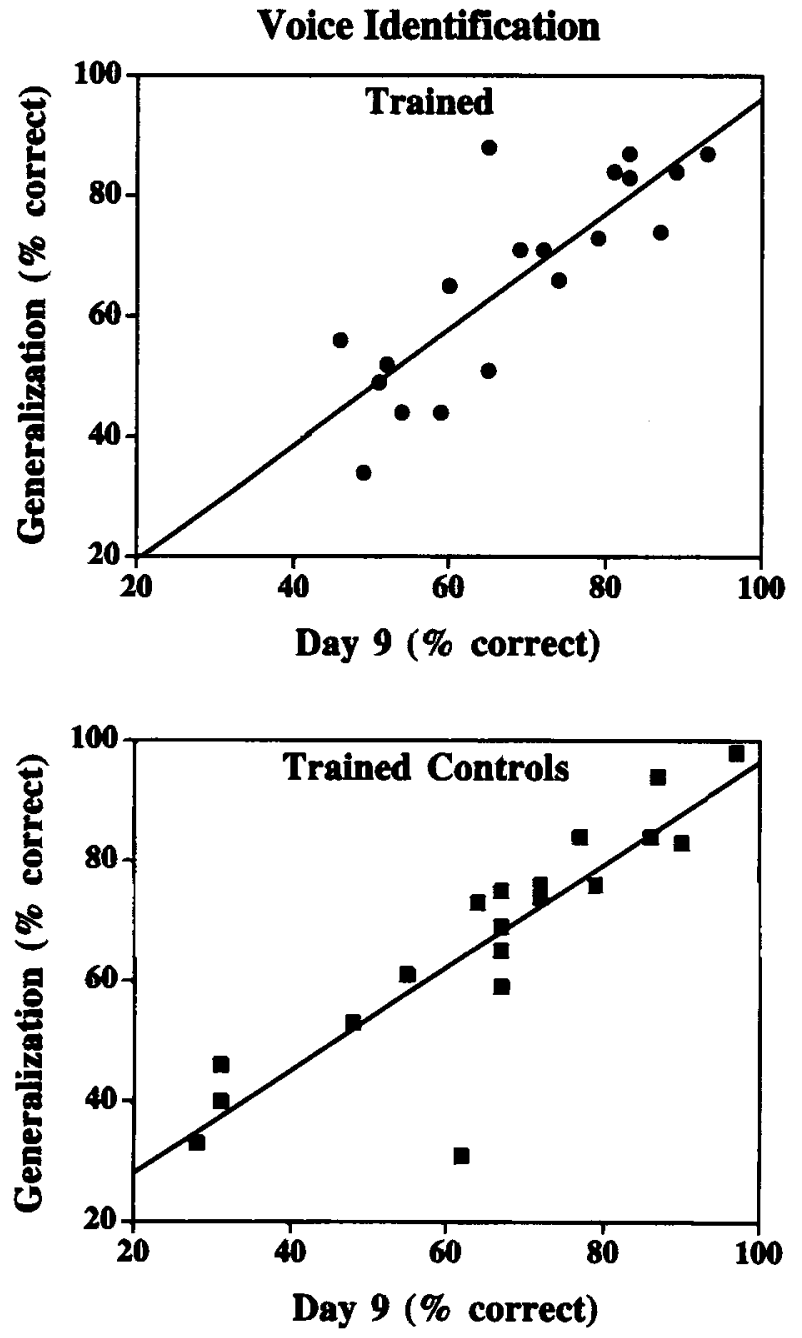

Figure 1. Scatterplots of percent correct voice identification for each listener on Day 9 are plotted as a function of percent correct voice identification for the generalization test on Day 10. The top panel shows the results for the trained experimental group and the bottom panel shows the results for the trained control group.

test given in the 10th session. The results displayed in this figure and in the subsequent training figures were always drawn from the final test with no feedback that was given on each day of training. The top graph shows the data from the listeners in the experimental group, and the bottom graph shows the data from the listeners in the control group, averaged across talkers. Recall that both groups of listeners received identical training with the same group of talkers. The stimulus set used for the subsequent word intelligibility test distinguishes these groups. This figure illustrates two aspects of our results. First, performance on the 9th day of training is well correlated with performance in the generalization test with novel words $[r(17)=$ $+.83, p<.01$, for the experimental group; $r(17)=+.88$, $p<.01$, for the control group]. Second, for both groups of listeners, individual subjects differed greatly in per- 
formance. The range on Day 9 was 69 percentage points from the poorest to the best learner.

Because of the large individual differences, the listeners were divided into two groups on the basis of their voice identification scores. ${ }^{l}$ A criterion of $70 \%$ correct voice identification on the 9th day of training was selected to group them into "good" and "poor" learners. Our rationale for dividing our listeners into two groups was that to assess the effects of voice learning on word intelligibility, we needed to have a group of listeners who had indeed learned the set of voices used in the experiment. This partitioning of the data on the basis of this performance criterion also allowed us to compare listeners who successfully learned the voices with listeners who did not become as sensitive to the individual characteristics of each voice. With this criterion, 9 subjects from both the experimental and the control conditions were classified as "good" learners, ${ }^{2}$ and 10 subjects from both the experimental and the control conditions were classified as "poor" learners.

Figure 2 shows the listeners' voice identification performance, averaged across talker's voice, for the test phase of Days 1-9 of training and for the generalization test on Day 10 . Percent correct voice identification is plotted as a function of day of training for "good" and "poor" learners in both the experimental and the control conditions. Again, recall that the experimental and the control groups were given training on the same set of voices. All subjects identified talkers consistently above chance even on the lst day of training, and all listeners improved over the 9 days of training. A three-way repeated measures analysis of variance (ANOVA) with training group (experimental vs. control), day of training (Days 1-9 and generalization), and listener learning group ("good" vs. "poor") as factors was conducted on the percent of correct responses. A significant main effect of days of training was found $[F(9,306)=69.58, p<.001]$, indicating that overall, the listeners' voice identification performance improved over days of training. A significant main effect of learning group was also found $[F(1,34)=78.31, p<$ $.001]$, indicating that "good" learners identified talkers" voices more accurately than "poor" learners. In addition, a significant interaction between days of training and learner group was found $[F(9,306)=9.55, p<.001]$. "Good" learners improved to a greater extent over days of training than did "poor" learners. From Day 1 to Day 9 , "good" learners' performance rose from $46.06 \%$ to $81.72 \%$ correct, while "poor" learners performance rose only from $38.9 \%$ to $54.5 \%$ correct.

\section{Perceptual Spaces for Voices}

In order to examinc the perceptual spaces for these voices and how they changed over time with training, multidimensional scaling (MDS) was performed on the confusion matrices generated during the first and last day of training for "good" and "poor" listeners. The matrices were constructed by using the number of times listeners confused each voice with each of the other nine voices during the test phase administered to listeners at the end of the first and ninth session of training. Four separate three-dimensional scaling solutions were calculated (see Nygaard \& Kalish, 1994) for each of the four day versus

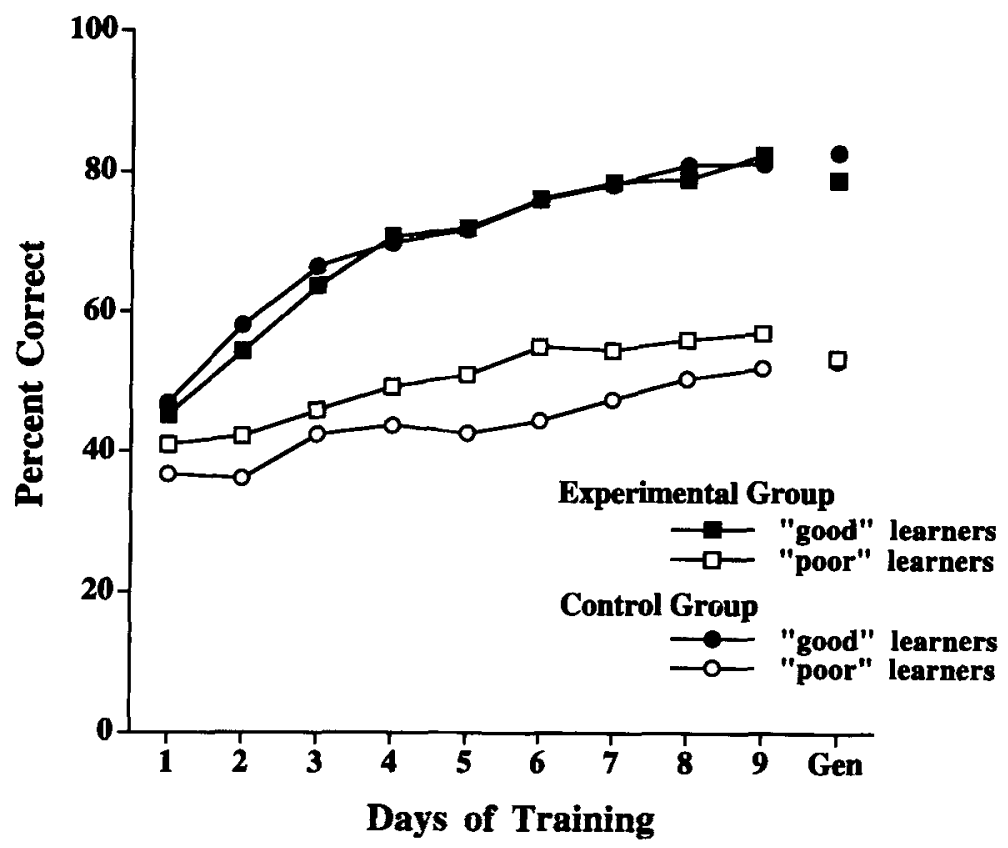

Figure 2. Percent correct voice identification from isolated words is plotted for each day of training and for the generalization test for both "good" and "poor" learners in the trained experimental and control groups. 
"Poor" Learners

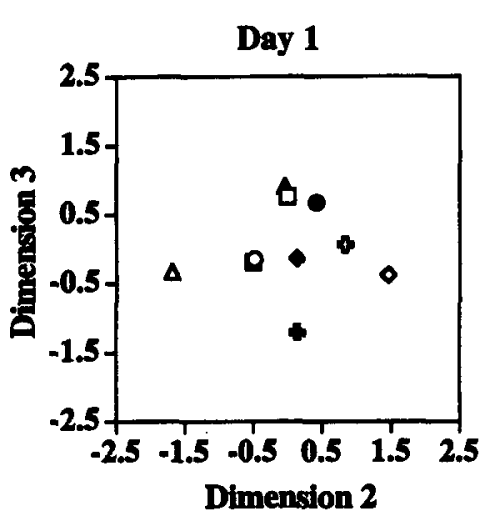

"Good" Learners

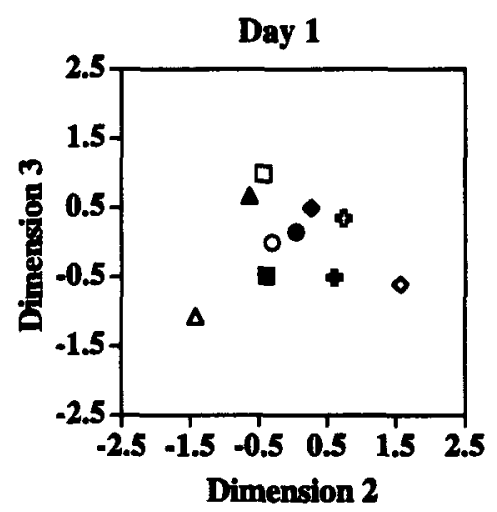

口 $\triangle \oplus \diamond \circ$ Males

- $\Delta+\bullet \bullet$ Females
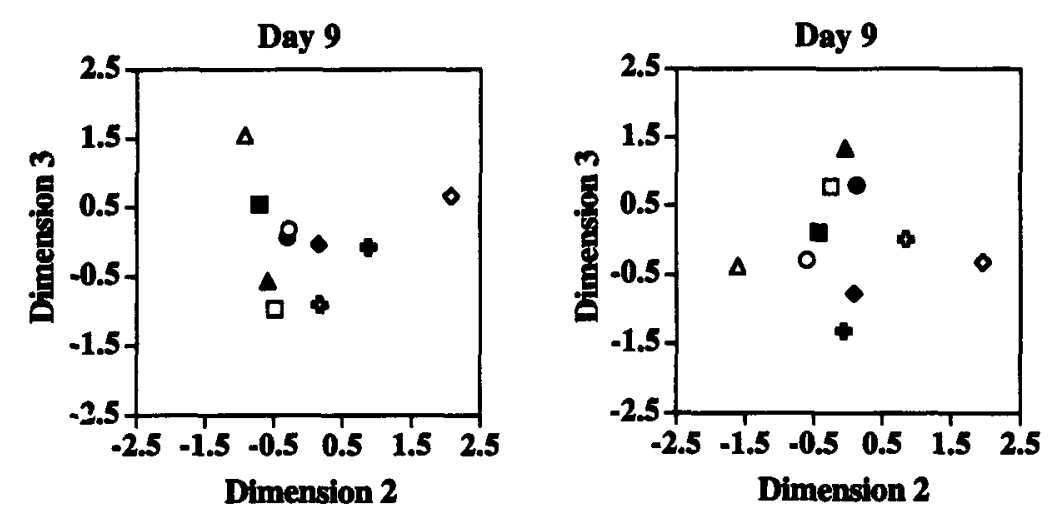

Figure 3. Dimensions 2 and 3 of multidimensional scaling solutions are plotted for Day 1 of training (top panels) and Day 9 of training (bottom panels). Scaling solutions for the "poor" learners are on the right, and solutions for the "good" learners are on the left.

group combinations. Figure 3 shows two-dimensional (Dimensions 2 and 3) representations of each of the four solutions. Dimension 1 is not represented in this figure, because, across all solutions, it uniformly corresponded with sex of the speaker. Although Dimensions 2 and 3 do not map onto obvious acoustic dimensions of the speech signal, the differences in perceptual distances among talkers for "good" and "poor" listeners is diagnostic. For both "good" and "poor" learners, there is a considerable amount of perceptual confusion on the first day of training. Correlations between MDS coordinates for "good" and "poor" learners across both male and female talkers are significant for each dimension after the first day of training $[r(8)=+.99, p<.01$, for Dimension $1 ; r(8)=$ $+.92, p<.01$, for Dimension $2 ; r(8)=+.72, p<.02$, for Dimension 3]. However, "good" and "poor" learners also differ after the last day of training. Correlations between coordinates for "good" and "poor" learners were not significant for Dimension 3 on Day 9 of training $[r(8)=$ $+.98, p<.01$, for Dimension $1 ; r(8)=+.83, p<.01$, for Dimension $2 ; r(8)=-.39$, n.s., for Dimension 3]. Male and female talkers are well separated in perceptual space for the "good" learners, but there is no such separation for the "poor" learners. For "good" learners, male speakers are well represented along Dimension 2 and female speakers are well represented along Dimension 3. For "poor" learners, female speakers are not separated along either Dimension 2 or Dimension 3.

The results of the scaling solutions also illustrate the differences in identifiability of the voices used in training. Individual talkers' voices were quite different in how easily they could be learned by listeners. Figure 4 shows scatterplots of Day 9 talker identification performance, plotted against generalization test identification scores for individual talkers, averaged across listeners. The top graph shows data from the listeners in the experimental group, and the bottom graph shows data from the listeners in the control group. This figure illustrates two aspects of the learning data. First, individual talker identification on Day 9 of testing is significantly correlated with performance on the generalization test for both groups $[r(8)=+.89, p<.01$, for the experimental group; $r(8)=$ 

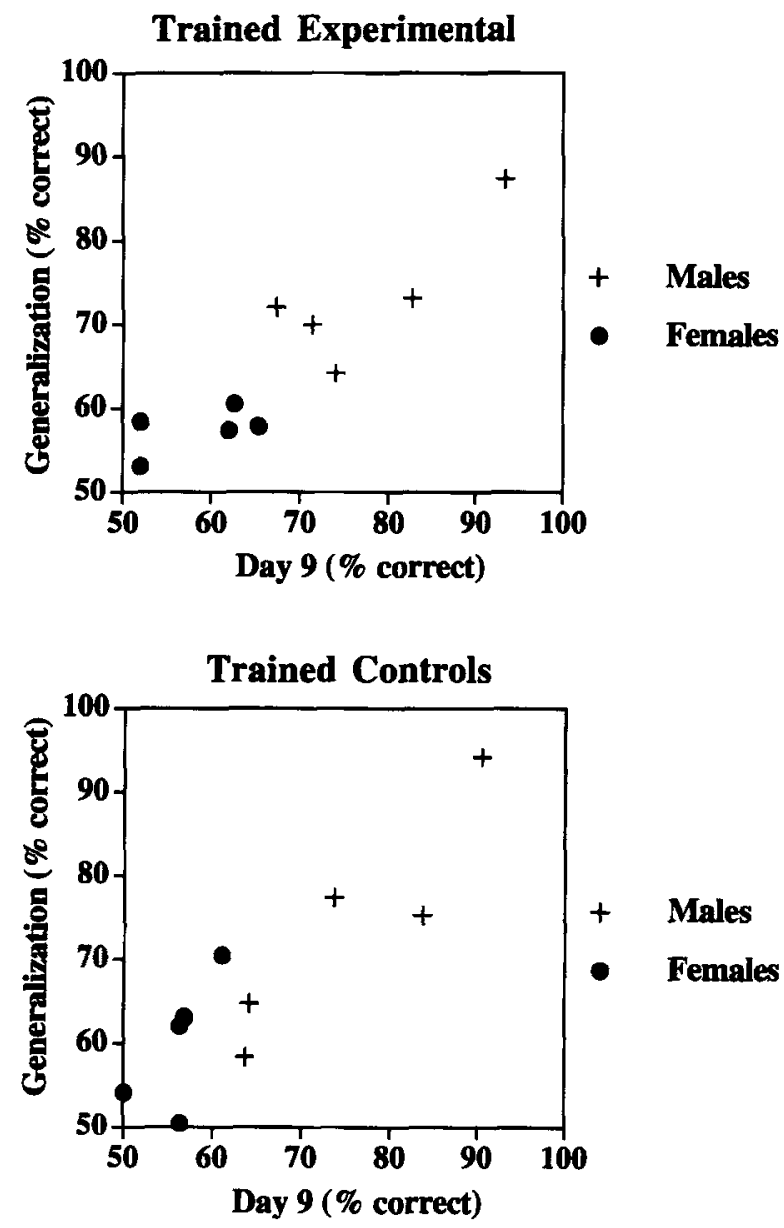

Figure 4. Scatterplots of percent correct voice identification performance for each individual talker (males and females) on Day 9 is plotted as a function of percent correct voice identification on generalization test. The top panel shows the results for the trained experimental group, and the bottom panel shows the results for the trained control group.

$+.91, p<.01$, for the control group]. Second, for both groups of listeners, identification performance varied greatly, depending on the individual voice. For example, identification scores for male voices were superior to identification scores for female voices, at least for the set of voices used in this experiment.

\section{Generalization}

The generalization test showed recognition of voices from the novel words presented on Day 10 almost identical to that on the final day of training. These results are also shown in Figure 2. In the experimental condition, percent differences between the generalization test and Day 9 of training were 3.55 and 3.66 for "good" and "poor" learners, respectively. In the control condition, percent differences between the generalization test and Day 9 of training were 1.89 and 1.00 for "good" and "poor" learners, respectively. $t$ tests revealed no significant differ- ences in listeners' performance across conditions between the two tests.

\section{Word Intelligibility}

Figure 5 shows percent correct word identification as a function of signal-to-noise ratio for both groups of trained listeners and for both groups of untrained listeners. The top graph shows data from the "good" learners, and the bottom graph shows data from the "poor" learners. Two separate repeated measures ANOVAs were conducted for the "good" and "poor" learners, using training condition (trained experimental, trained, and untrained controls) and signal-to-noise ratio $(+10,+5,0,-5)$ as factors. The data from all the listeners in both untrained control groups were used as a comparison for both "good" and "poor" learners, and the same data are included in both analyses and in both panels of Figure 5 .

"Good" learners. The analysis for the "good" learners revealed a significant main effect of signal-to-noise ratio $[F(3,126)=351.55, p<.001]$. As expected, identification performance decreased from the +10 to the -5 signal-to-noise ratio for all four groups. The analysis also revealed a significant main effect of training condition $[F(3,42)=7.43, p<.001]$, indicating that identification performance differed across the four training conditions. A significant interaction was found between training condition and signal-to-noise ratio $[F(9,126)=$ $3.03, p<.001]$, suggesting that identification performance among the groups was larger at some signal-tonoise ratios than at others.

A post hoc Tukey HSD analysis was conducted for pairwise comparison of the means. The trained experimental group was found to differ significantly $(p<.05)$ from the trained control group as well as from the two untrained groups. No significant differences were found in the pairwise comparisons of the trained and untrained control groups. This analysis confirms that the significant main effect for training condition found in the original study by Nygaard et al. (1994) was due to better word identification performance in the trained experimental group, who received words produced by familiar voices at test, than in the three control groups, who received words produced by unfamiliar voices at test.

"Poor" learners. The analysis for the "poor" learners also revealed a significant main effect of signal-to-noise ratio $[F(3,132)=290.85, p<.001]$, indicating that identification performance decreased from the +10 to the -5 signal-to-noise ratio for all four groups. No main effect of training group $(p>.73)$ or interaction between training group and signal-to-noise ratio $(p>.14)$ was found. Word identification performance across training groups did not differ significantly.

\section{Discussion}

Several important findings were obtained in Experiment 1 . First, listeners displayed large individual differences in their ability to learn to identify a set of 10 voices 

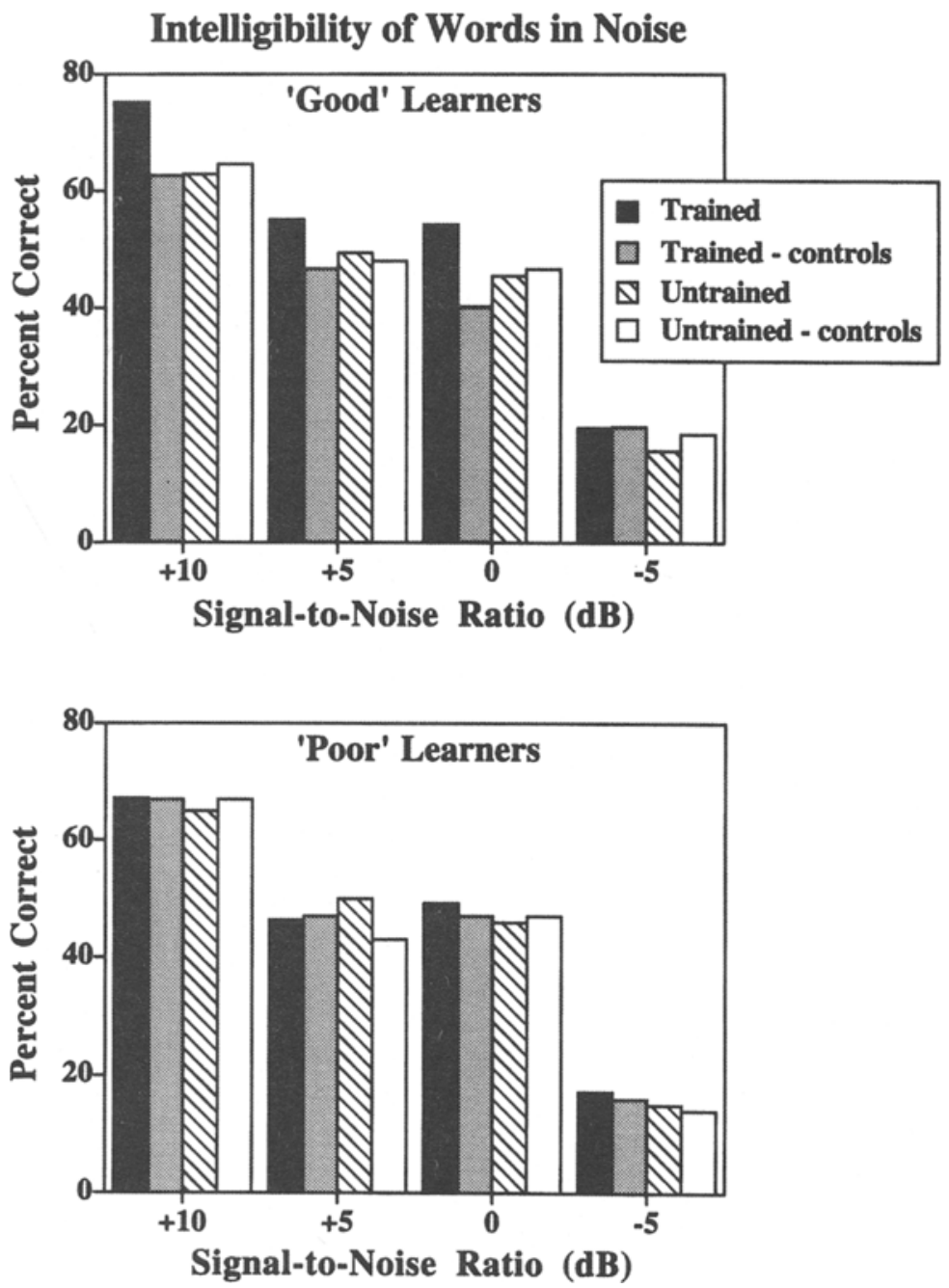

Figure 5. Percent correct word recognition for both training groups (experimental and control) and both untrained groups is plotted for each signal-to-noise ratio. The top panel shows results for the "good" learners, and the bottom panel shows results for the "poor" learners.

from isolated words. Individual listener performance across training groups ranged from $28 \%$ correct for the poorest learner to $97 \%$ correct for the best learner, after 9 days of training. This finding suggests that simple exposure to the set of voices over the 9-day period was not sufficient for perceptual learning of talkers' voices to occur.

Second, given that these listeners differed in their ability to learn the voices, it was possible to characterize some of the listeners as "good" voice learners and others as "poor" voice learners. Although voice learning performance represents a continuum of ability (see Figure 1), grouping the listeners in this manner provided a useful heuristic for analyses of the consequences of different learning abilities. For example, we found that "good" and "poor" learners differed not only in their absolute ability to identify voices by Day 9 , but also in the amount of learning that took place over time. That is, "good" and "poor" learners started out as very similar at the end of the 1 st day of training ( $t$ tests revealed no significant difference between groups on Day 1), but their identification performance quickly diverged over the next 9 days of training. "Good" learners improved to a much greater extent than did "poor" learners. This divergence suggests that through practice in categorizing and explicitly identifying voices, "good" learners become "attuned" to the fine acoustic-phonetic details that distinguish each talker's voice. "Poor" learners do not seem to acquire the same kind of perceptual sensitivity using these voice dimensions during this type of laboratory training task.

Additional clues to the differences between the two groups come from our MDS analyses. "Good" and "poor" learners appear to have developed qualitatively different perceptual strategies to identify different talkers, which may account for their ultimate success in the voice recog- 
nition task. Although both groups appeared to distinguish male from female voices, they differed in the other dimensions that they used to discriminate individual female and individual male talkers. By the end of the 9th day of training, the "good" learners appeared to use Dimension 3 to distinguish female talkers and Dimension 2 to distinguish male talkers. The "poor" learners appeared to be using both dimensions to discriminate all the talkers, and this difference in strategy may account for the differences in identification performance. Of course, a note of caution should be mentioned here. These scaling solutions only provide a suggestion of strategic differences in voice learning, and no acoustic dimensions have been identified that are related to the dimensions that result from scaling. In addition, it should be noted that the "poor" learners did improve somewhat over the 9 days of training and, presumably, could eventually have learned to identify our set of talkers to criterion if the training had been extended in time. Given the limitations of the study, it was not possible to determine whether "poor" learners, or "good" learners for that matter, would continue to improve with additional training or whether "poor" learners would eventually switch to a more optimal learning strategy. This is obviously a question for future research.

The individual differences found among listeners is complemented by individual differences in the identifiability of talkers' voices. Both the scaling analyses and performance differences in identifying individual talkers' voices suggest that talkers vary a great deal in their perceptual distinctiveness. In particular, it appears that male voices, at least those used in this experiment, were significantly easier to identify than the female voices (see also Thompson, 1985). In addition, whatever makes each voice more or less perceptually distinctive in terms of ease of identification is at least somewhat abstract with respect to the specific items used during training. Listeners were quite good at generalizing what they had learned to a new set of stimulus materials in the talker generalization task. Listeners were able to use talker-specific knowledge to identify voices from linguistic tokens that they had never been exposed to before, suggesting that listeners learned something more general about each talker's voice and style of speaking. Thus, it appears that both talker-specific and listener-specific variables contribute to the eventual identification of a talker's voice (Bradlow, Nygaard, \& Pisoni, 1995).

Finally, the most important finding to emerge from this study is that familiarity with a talker's voice influences linguistic processing, and specifically, the intelligibility of isolated words mixed in noise. Perceptual learning of a set of novel talkers' voices caused listeners to be better able to recover the linguistic content of the signal. This finding marks one of the first experimental demonstrations that the perceptual mechanisms responsible for analyzing talker identity are not independent from the mechanisms responsible for extracting the lexical content of an utterance from the speech wave form.
Perceptual learning and long-term retention of talkers' voices selectively modified the ability of listeners to process the phonetic content of these speech signals.

The present results also demonstrate that through learning to associate a name with each talker's voice, listeners began to attend to talker-specific aspects of the speech signal that were also relevant for perceiving the linguistic content of the same signal. The perceptual dimensions related to talker identity appeared to become much more distinctive during categorization training, and this perceptual sensitivity transferred to the processing of linguistic information. This transfer of learning or sensitivity from talker identity to word recognition is crucial, because it implies that these two sources of information, and the perceptual processing of these two sets of dimensions, are inexorably linked in speech perception. In a more general sense, talker identity and linguistic information appear to be integral dimensions analogous to color dimensions such as brightness and saturation (Goldstone, 1994). Although lexical and indexical information are arguably higher order aspects of spoken language, they may nevertheless behave like lower level perceptual dimensions (see Mullennix \& Pisoni, 1990).

The differences in performance between the "good" and "poor" learners in this experiment indicate that associative learning was a necessary but not sufficient condition for listeners to learn each talker's voice and consequently for listeners to show a benefit of training with talker identity on word recognition. Although all listeners received the same amount of training, only listeners who could successfully identify the talkers' voices explicitly showed a benefit in the word recognition test. This indirect test of the type of perceptual learning necessary for word intelligibility to be affected provides evidence for the assertion that mere exposure or mere repetition of the voices over a period of time does not result in sufficient perceptual differentiation along the voice dimension to modify processes of spoken word recognition (see E. J. Gibson, 1969). One explanation of these results is that the "poor" learners did not receive sufficient training to "fine tune" or adjust their attentional mechanisms to the relevant talker-specific information in the signal. For whatever reason, the "good" learners were able to attend to the specific acoustic-phonetic details that not only reliably distinguished one talker's voice from another but also reliably helped in processing the phonetic aspects of the speech signal. It should be noted that the "poor" learners did not necessarily have difficulty processing speech from a variety of talkers, but rather, when the perceptual system was taxed, as when words were presented in noise, they were unable to utilize their prior knowledge of each talkers' idiosyncratic style of speech to help recover the phonetic content and lexical information in the signal.

Although we proposed that attentional differences between the "good" and "poor" groups of learners account for both the differences in perceptual learning of voice and their ability to identify linguistic aspects of the signal 
produced by "pre-exposed" talkers, we have no direct evidence that it is attention during learning to talker-specific details that results in perceptual sensitivity for linguistic information as well. In the next two experiments, we addressed this issue more directly by attempting to experimentally focus listeners' attention on specific aspects of voice information and then evaluating how well listeners generalized this specific learning to a linguistic task. By specifically manipulating the type of talker information available during training and then evaluating linguistic processing for matched or mismatched material, we could evaluate how perceptual learning of talker identity might be related to perceptual sensitivity for linguistic information encoded in the speech signal.

\section{EXPERIMENT 2}

Experiment 2 was designed to assess the nature and extent of the perceptual learning demonstrated in the first experiment. To that end, listeners were trained to recognize a set of 10 talkers from sentence-length rather than from word-length utterances. After training was completed, intelligibility was assessed with the use of isolated words produced by familiar and unfamiliar talkers. The aim of this study was to determine whether the information learned about a talker's voice from sentences generalizes to the perception of isolated spoken words. The assumption was that training with sentence-length utterances would focus listeners' attention at a different level of analysis than does training with isolated words. It was hypothesized that because sentences contain highly distinctive prosodic and rhythmic information in addition to the specific acoustic-phonetic implementation strategies and physiological characteristics unique to individual talkers, perceptual learning of voices from sentences would require attentional and encoding demands specific to those test materials.

Two groups of listeners learned to identify voices from sentence-length utterances over a 3-day training period. The experimental group was then tested with isolated words mixed in noise to assess intelligibility of talkers they had been exposed to in training. The control group was tested with isolated words produced by a set of unfamiliar talkers. The isolated words used at test also differed in their lexical characteristics. Half were "easy words"-high-frequency words from sparse lexical neighborhoods - and half were "hard words"-low-frequency words from dense lexical neighborhoods (Luce, Pisoni, \& Goldinger, 1990). Because lexically hard words require attention to fine acoustic-phonetic detail for successful lexical access, it was hypothesized that perceptual learning of talkers' voices might improve the identification of lexically hard words presented in noise to a greater extent than lexically easy words.

\section{Method}

\section{Listeners}

The subjects were 46 undergraduate and graduate students at Indiana University. Twenty-seven listeners served in the experimen- tal condition, and 19 served in the control condition. All listeners were native speakers of American English and reported no history of a speech or hearing disorder at the time of testing. The listeners were paid for their participation.

\section{Stimulus Materials}

Two sets of stimuli were used in this experiment. The sentence training stimuli consisted of 100 Harvard sentences (Egan, 1948; IEEE, 1969) produced by 10 male and 10 female talkers. These sentences are all meaningful English, monoclausal sentences containing $5 \mathrm{key}$ words plus a variable number of function words. The key words all contained one or two syllables. The isolated word stimuli consisted of 100 monosyllabic words produced by 10 of the same talkers ( 5 male and 5 female) who produced the sentence materials. None of the isolated words were used in the sentence stimuli. The isolated words varied in their neighborhood characteristics (Luce et al., 1990). Fifty "easy" and 50 "hard" words were selected. "Easy" words were high-frequency items that were selected from sparse lexical neighborhoods. "Hard" words were low-frequency words that were selected from dense lexical neighborhoods. A lexical neighborhood consists of the set of words which differ by one phoneme from the target word (Luce et al., 1990). In addition, all of the isolated words were rated as highly familiar (Nusbaum et al., 1984). All stimuli were digitized on line at a sampling rate of $20 \mathrm{kHz}$ using 16-bit resolution. The RMS amplitude levels for all stimuli were digitally equated.

\section{Procedure}

Two groups of listeners completed three training sessions with sentence-length materials. The digitized stimuli were presented with the use of a 16-bit digital-to-analog converter and were lowpass filtered at $10 \mathrm{kHz}$. The stimuli were presented to the listeners over matched and calibrated TDH-39 headphones at approximately $80 \mathrm{~dB}$ SPL. A pretest-posttest design was used in which both groups of listeners received identical pre- and posttests with isolated words produced by the same set of talkers. Each group was then trained, using different sets of talkers. For the experimental group, the same talkers were used for pre- and posttests and for training. For the control group, different talkers were used during training than in the pre- and posttests. Thus, in this experiment, we were able to directly compare word intelligibility performance for the same set of words before and after training.

Pretest word intelligibility. In both the pretest and posttest, 100 isolated words produced by 10 talkers ( 5 male and 5 female) were presented at $80,75,70$, or $65 \mathrm{~dB}$ (SPL) mixed in continuous white noise that was low-pass filtered at $10 \mathrm{kHz}$ and presented at $70 \mathrm{~dB}$ (SPL) over matched and calibrated TDH 39 headphones. This manipulation yielded four signal-to-noise ratios: $+10,+5,0$, and $-5 \mathrm{~dB}$. Equal numbers of words were presented at each of the four signalto-noise ratios. The listeners were asked to identify each word by typing their response on a keyboard. The responses were recorded on line by a PDP-11/34 computer.

Training. The two groups of listeners also completed 3 days of training in order to familiarize themselves with the voices of 10 talkers. As in Experiment 1, both groups were required to identify each talker's voice and associate that voice with one of 10 common names on each day of training. Both groups of listeners completed three different tasks: familiarization, recognition, and testing. The tasks as well as all other aspects of training were identical to those in Experiment 1.

Posttest word intelligibility. After 3 days of sentence training, the listeners received a posttest word recognition test identical to the pretest. They were asked to identify the linguistic content of isolated words produced by familiar or unfamiliar talkers at four signal-tonoise ratios.

Generalization. After the posttest, the experimental group received a generalization test in which the set of words used in the preand posttests was presented to listeners for voice identification. The 
listeners were asked to identify the talker (rather than the word) on each trial from the same isolated words that had been used in the posttest. This test allowed us to determine how well the perceptual learning of voices from sentences generalized to identification of voices from isolated words.

\section{Results}

\section{Training}

As in the first experiment, we found individual differences in listeners' voice identification performance. However, far fewer listeners failed to reach the criterion performance of $70 \%$ correct on the 3rd day of training when they were required to learn voices from sentences. Because there were too few "poor" listeners, particularly in the control condition, for separate "good" and "poor" learner analyses, the 11 subjects from the experimental condition and the 2 subjects from the control condition who failed to reach criterion were simply eliminated from the overall analysis. That left 16 subjects in the experimental conditions and 17 subjects in the control condition.

Figure 6 shows voice identification performance for the experimental and control groups over the 3 days of training. All listeners showed continuous improvement over the 3 days of training. Both groups identified talkers consistently above chance even on the 1st day of training, and performance rose to nearly $85 \%$ correct by the last day of training. A repeated measures ANOVA with learning and days of training as factors showed a significant main effect of day of training $[F(2,62)=74.04, p<$ $.001]$ and also a significant main effect of group $[F(1,31)=$ $20.27, p<.001]$. The control group performed significantly better than the experimental group in learning their set of talkers.

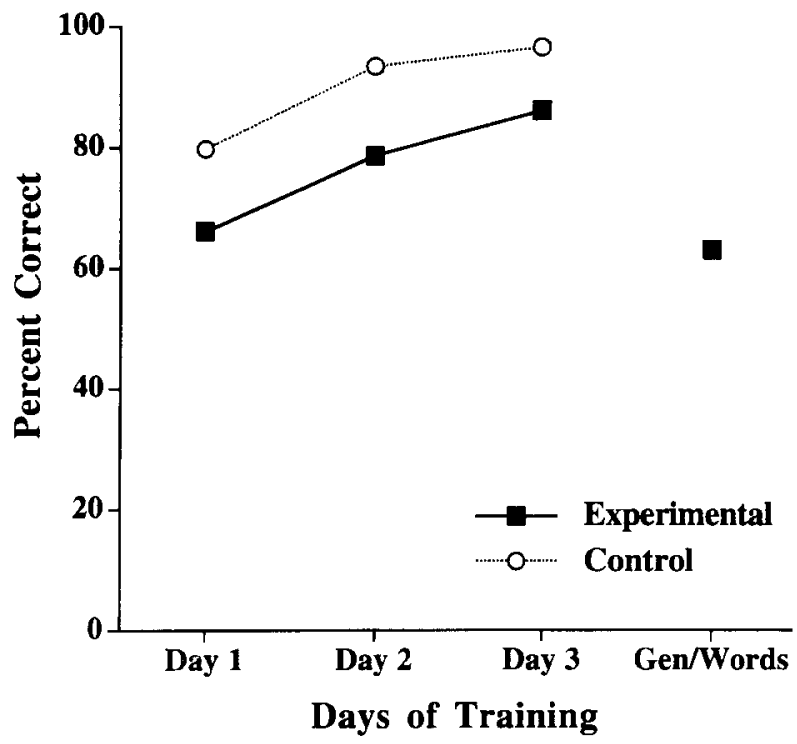

Figure 6. Percent correct voice identification from sentences is plotted for each day of training and for the generalization test given to the experimental group.
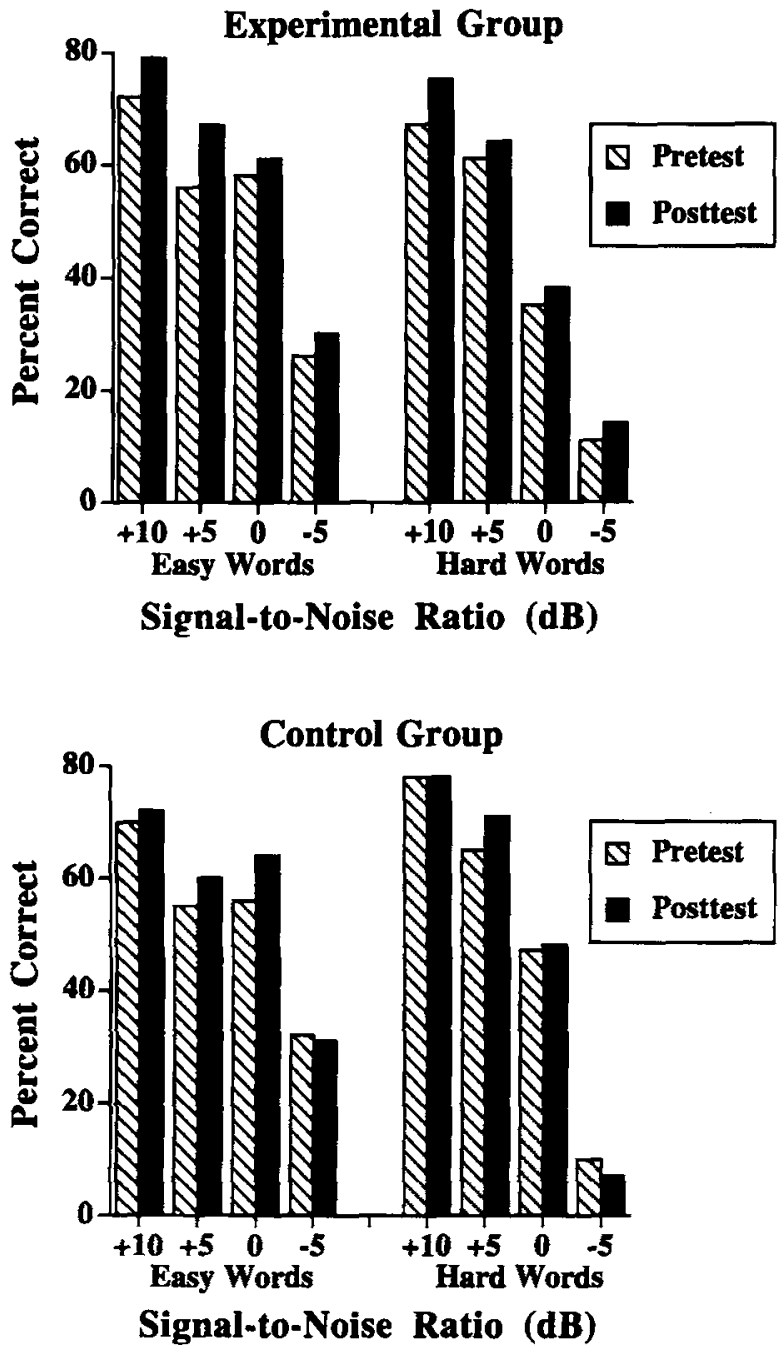

Figure 7. Percent correct pre- and posttest word recognition performance is plotted at each signal-to-noise ratio for easy and hard words. The top panel shows results for the experimental group, and the bottom panel shows results for the control group.

\section{Generalization}

Figure 6 also shows voice identification performance on the word generalization test. Recall that the set of isolated words used at test comprised familiar voices only for the experimental group. The listeners in the experimental group were $63 \%$ correct in the word generalization task at identifying the voices that they had learned during training from sentences. This level of performance was not significantly different from voice identification performance on sentences at the end of the 1st day of training.

\section{Isolated Word Intelligibility}

Figure 7 shows percent correct word identification at pretest and posttest as a function of signal-to-noise ratio and lexical neighborhood structure. The top panel shows the results for the experimental group, and the bottom 
panel shows results from the control group. A four-way repeated measures ANOVA with training group (experimental vs. control), test (pre- vs. posttest), word type ("easy" vs. "hard"), and signal-to-noise ratio $(+10,+5,0$, -5 ) as factors was calculated on percent correct responses. The analysis revealed main effects of signal-to-noise ratio $[F(3,93)=408.30, p<.01]$, test $[F(1,31)=19.73, p<$ $.01]$, and word type $[F(1,31)=55.41, p<.01]$, reflecting an influence of signal-to-noise ratio on responding, superior post- versus pretest performance, and better performance with "easy" than with "hard" words. There were two significant two-way interactions: one involving word type and condition $[F(1,31)=8.54, p<.05]$ and one involving word type and signal-to-noise ratio $[F(3,93)=$ $39.05, p<.01]$. Both interactions reflect differences in the intelligibility of "easy" and "hard" words depending on experimental group and signal-to-noise ratio. Finally, one significant three-way interaction involving word type, signal-to-noise ratio, and condition was found $[F(3,93)=$ $4.77, p<.05]$, reflecting differences in the pattern of performance on "easy" versus "hard" words as a function of signal-to-noise ratio and experimental condition. No other main effects or interactions were significant.

Because the overall ANOVA confirmed that posttest performance was superior to pretest performance, presumably owing to mere repetition of the same items, additional analyses were conducted using the magnitude of the difference between pre- and posttest performance for the experimental versus the control groups. To assess the effects of perceptual learning on word intelligibility, the difference in percent correct word identification from pretest to posttest was calculated for each listener. Figure 8 shows these difference scores for both the experimental and the control groups, averaged across signalto-noise ratio, for both "easy" and "hard" words. Although there was greater improvement for subjects in the experimental condition who heard the familiar voices at posttest than for subjects in the control condition, the effects of voice familiarity on word intelligibility were small and did not reach statistical significance $[F(1,31)=3.33$, $p<.08]$. A repeated measures ANOVA calculated on the difference scores averaged across signal-to-noise ratio with training group (experimental versus control) and word type ("easy" vs. "hard") as factors showed no significant main effects or interactions.

\section{Discussion}

These findings demonstrate that perceptual learning of voices improves dramatically as longer duration utterances are used to familiarize listeners with those voices. The majority of listeners in this experiment learned to identify talkers' voices over three training sessions instead of the nine training sessions needed in the first experiment. Further, a larger percentage ( $72 \%$ for sentences vs. $47 \%$ for words) of listeners achieved a criterion of $70 \%$ voice identification when learning voices from sentencelength utterances even with fewer days of training. There are at least two explanations for improved learning with sentence-length utterances. One is that sentence-length utterances merely provide listeners with a larger sample of speech containing the same information that they get with word-length utterances (Peters, 1955a). The reason why listeners are better able to identify voices from sentencelength utterances is that they receive, in effect, five or so words on which to make their talker identity judgments on each trial rather than just one word which they received when training with isolated words. A second explanation, however, is that sentence-length utterances also contain additional, qualitatively different information. That is, sentence-length utterances may provide listeners both with

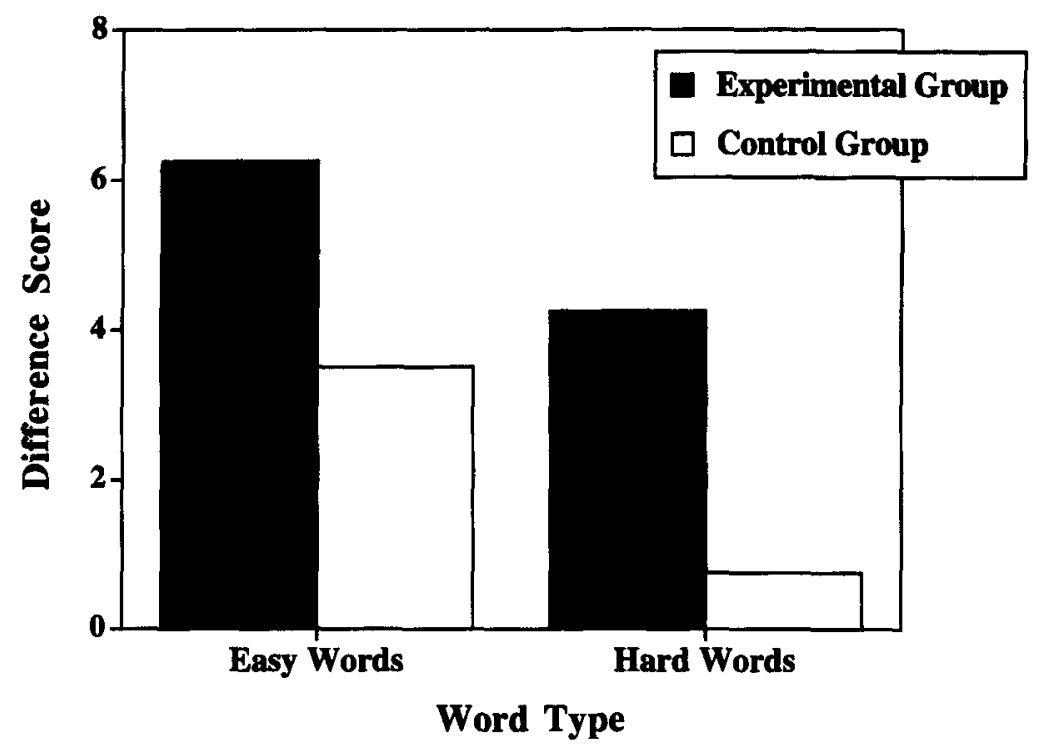

Figure 8. Percent difference scores collapsed across signal-to-noise ratio are plotted for word type and experimental group. 
the voice-specific information found in word-length utterances and with other sources of information about fundamental frequency, duration, and rhythm, which vary over the entire utterance. Although word-length utterances may also contain prosodic information, it is assumed that the sentence-length utterances used in this experiment may have had a more varied prosodic and intonational pattern. Thus, when listening to sentences, the subjects were exposed to prosodic and rhythmic information specific to the sentence level in addition to the acoustic-phonetic implementation and intonational differences among talkers at the word and segmental levels.

The results of the generalization test suggest which explanation might account for the differences in learning rates between training with words and training with sentences. Generalization of learning using sentences to isolated words was not very good. It appears that listeners learned a qualitatively different set of acoustic properties to identify talkers from sentence-length utterances than they did to identify them from isolated words. Although it could be argued that the listeners in this experiment received far less training on the set of voices, it is still the case that they were identifying talkers' voices quite well by Day 3 from sentence-length utterances. This finding suggests that in learning to identify voices from sentences, listeners are allocating their attention to different attributes of the signal and several different levels of analysis. In this task, they are not required to attend as closely to the fine acoustic-phonetic details that distinguish voices at the word level. Rather, listeners are learning to distinguish voices along perceptual dimensions in the sentence-length utterances that do not completely overlap with the dimensions used to distinguish voices at the word-length level.

Given that learning voices from sentences does not generalize well to the perception of talker identity in isolated words, it is not surprising that this perceptual learning does not significantly affect the intelligibility of isolated words. Although listeners who heard familiar voices in the posttest were somewhat better at identifying novel isolated words than were listeners who heard unfamiliar voices, these results fell just short of significance. The listeners appeared to focus on a talker-specific dimension in the sentence-length utterances that was not as useful in the word-length utterances. If this account is true, however, listeners should show large effects of talker identification training with sentence-length utterances on the intelligibility of sentences. Thus, if there is a match between the type of talker information that is learned during training and the type of linguistic information that is presented at test, then perceptual learning of voices should once again strongly influence the perception of the linguistic attributes of the signal. Experiment 3 was designed to address this issue.

\section{EXPERIMENT 3}

Experiment 3 was similar to the second experiment, except that after the training of listeners to learn talkers' voices from sentence-length utterances was completed, subjects were given an intelligibility test using sentences produced by familiar and unfamiliar talkers. Two questions were addressed here. First, does specific training on sentence-length utterances generalize to similar test materials? We predicted that the talker-specific information learned from sentences would influence the recognition of words in sentences. Therefore, when a match between information learned during training and information required at test occurred, we expected that the transfer of perceptual learning along the talker identity dimension would increase perceptual sensitivity to the linguistic content, as had been found in the first experiment.

Second, are sentence-length utterances that have semantic and syntactic constraints susceptible to the effects of familiarity with a talker's voice? This experiment was also desıgned to determine whether talker-specific information could affect linguistic processing when other perceptual constraints might override its influence. Sentences not only contain the phonological and lexical information that influences the recognition of words, but they also contain higher-level syntactic and semantic constraints. Given the redundancy of linguistic information in sentences, our aim was to determine whether talker-specific information would influence the recognition of words in sentences or whether this source of information would become relatively unimportant in the context of sentences.

\section{Method}

\section{Listeners}

The subjects were 20 undergraduate and graduate students at Indiana University. Eleven listeners served in the experimental condition, and 9 served in the control condition. All listeners were native speakers of American English and reported no history of a speech or hearing disorder at the time of testing. The listeners were paid for their participation.

\section{Stimulus Materials}

Training and test stimuli were drawn from a digital database consisting of 100 Harvard sentences produced by 10 male and $10 \mathrm{fe}-$ male talkers (Bradlow, Torretta, \& Pisoni, 1996). Sentence identification tests showed greater than $90 \%$ intelligibility for all sentences in the quiet. Sentences were digitized on line at a sampling rate of $20 \mathrm{kHz}$, using 16-bit resolution. The RMS amplitude levels for all stimuli were digitally equated.

\section{Procedure}

Training. Training was similar to that in Experiments 1 and 2, except that the subjects were trained with a set of 50 sentences. Two groups completed the 3 days of training. The experimental group of 11 subjects learned the voices of the same 10 talkers who were used for the sentence intelligibility test. The control group of 9 subjects learned the voices of 10 different talkers. The listeners were not administered a pretest as in Experiment 2, because it was assumed that the set of 50 sentences used at test would be too memorable if used in a pretest as well.

Sentence intelligibility test. In the sentence intelligibility test, 48 novel sentences produced by 10 talkers ( 5 male and 5 female) were presented at 75,70 , or $65 \mathrm{~dB}$ (SPL) in continuous white noise that was low-pass filtered at $10 \mathrm{kHz}$ and presented at $70 \mathrm{~dB}$ (SPL), yielding three signal-to-noise ratios: $+5,0$, and $-5 \mathrm{~dB}$. An equal number of sentences was presented at each of the three signal-to- 


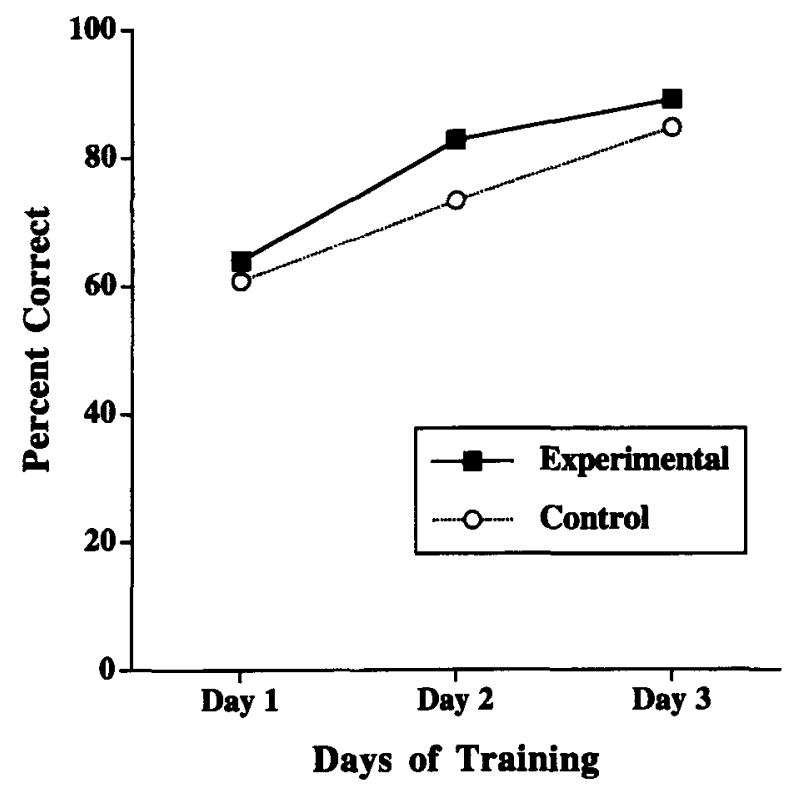

Figure 9. Percent correct voice identification from sentences is plotted for each day of training.

noise ratios. The subjects were asked to transcribe the sentence on a sheet of paper. For the subjects in the experimental condition, the sentences were produced by the 10 familiar talkers whom they had learned to identify during training. For the subjects in the control condition, the sentences were produced by 10 novel talkers whom they had not been exposed to during training.

\section{Results \\ Training \\ Figure 9 shows talker identification performance for the experimental and control groups over 3 days of train-}

ing. All subjects showed continuous improvement over the 3 days of training. As in Experiment 2, both groups of subjects identified talkers consistently above chance even on the 1st day of training, and performance rose to nearly $85 \%$ correct by the last day of training. A repeated measures ANOVA with learning and days of training as factors showed a significant main effect of day of training $[F(2,36)=78.029, p<.001]$, and no other significant effects.

\section{Sentence Intelligibility}

Transcription performance was scored in four ways for each sentence. The scoring methods were as follows.

Sentence correct. A response was scored as correct, if and only if the whole sentence was transcribed correctly (a sentence was still counted correct if the correct verb was used in the wrong form).

Keywords correct. The actual number of key words transcribed correctly out of five possible per sentence was scored.

Total words correct. The total number of words transcribed correctly per sentence was scored.

Meaning correct. A response was scored as correct when the sentence was correct (as stated above) or when the overall meaning of the sentence was correct.

Because all four scoring methods produced the same pattern of results, only the scoring for key words correct will be reported below.

Subjects' performance on the sentence intelligibility task was assessed by determining the number of key words correct in each test sentence, adding up the total number of correct key words across sentences and averaging these totals across subjects. Each Harvard sentence contained 5 key words, and the test set of 48 Harvard sentences contained 240 key words. Figure 10 shows the total num-

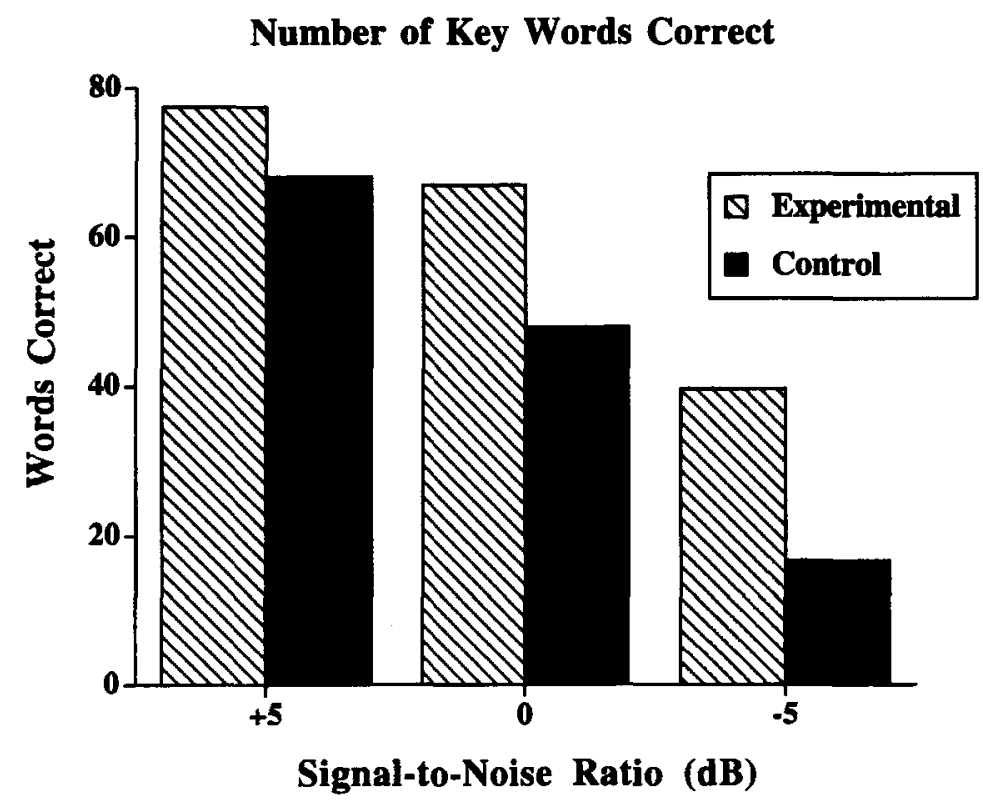

Figure 10. Number of key words correct is plotted as a function of signal-tonoise ratio for the experimental and control groups. 
ber of key words correct as a function of signal-to-noise ratio, averaged across subjects, for the experimental and control groups.

A repeated measures ANOVA with signal-to-noise ratio $(+5,0,-5)$ and training group (experimental vs. control) as factors showed a significant main effect of training group $[F(1,18)=220.378, p<.001]$. The subjects in the experimental condition who heard sentences produced by familiar talkers were able to transcribe more key words correctly across all signal-to-noise ratios than were the control subjects who heard sentences produced by unfamiliar talkers. A significant main effect of signal-to-noise ratio $[F(2,36)=286.26, p<.001]$ was also found, indicating better performance at the higher signal-to-noise ratios. Finally, there was a significant interaction between training group and signal-to-noise ratio $[F(2,36)=44.41$, $p<.001]$. As can be seen in Figure 8, this interaction demonstrates that the effect of talker familiarity became larger as signal-to-noise ratio decreased.

\section{Discussion}

These results demonstrate that perceptual learning of talkers' voices from sentences facilitates the recognition of words in sentences produced by familiar talkers. Learning talkers' voices from sentences generalized to the transcription of similar test materials, suggesting that through learning the distinctions among talkers during training, listeners became sensitive to talker-specific linguistic information that was relevant when perceiving sentences in noise. Listeners appear to attend to dimensions when learning voices from sentences that are most relevant when they must extract the linguistic content of sentence-length utterances. That is, perceptual learning in this task appears to involve attention to the specific dimensions of talker identity that are relevant at test. These findings replicate and extend the transfer of training results found in the first two experiments by demonstrating transfer of training from sentences to sentences, whereas none was found in Experiment 2 from sentences to words.

An interaction between familiarity and signal-to-noise ratio was also observed, suggesting that as listening conditions became more difficult, listeners made greater use of the talker-specific knowledge that they acquired in the first phase of the experiment. The difference between the experimental and control groups was larger at lower signal-to-noise ratios. Thus, as overall intelligibility of the stimulus set deteriorated, listeners were more likely to bring to bear talker-specific information to aid in their transcription performance. This finding suggests that listeners may use talker information to a greater extent in listening situations that are degraded. Familiarity with a talker's voice may be extremely important in most realworld listening situations. For example, these findings lead to the prediction that at cocktail parties, on city streets, and in other typical listening environments where there is noise or reverberation, listeners are better able to understand talkers whose vocal attributes are most familiar to them. These observations are consistent with clin- ical reports from hearing-impaired listeners who have difficulty with novel voices over the telephone or in noisy environments.

These results also confirm that learning to identify talkers' voices is much easier from sentences than from isolated words. As in Experiment 2, listeners readily learned to identify the 10 talkers over 3 days of training, and all listeners in this experiment reached our $70 \%$ criterion level of performance. This finding suggests that sentences are a rich source of talker-specific information and that learners are sensitive to the additional talker information in sentence-length utterances (Peters, 1955a, $1955 \mathrm{~b}$ ). Although we have not provided a direct test, it does appear that sentences provide qualitatively different sources of information about a talker's voice than do isolated words. That is, sentences appear to provide information about talker-specific acoustic-phonetic implementation strategies in addition to higher order information about idiosyncratic prosody, rhythm, and meter. During training, listeners apparently exploit all sources of information to help them learn the set of voices in this task.

Finally, the results confirm the importance of the role of talker information in spoken language processing. Familiarity with talkers' voice was found to affect the perception of sentence-length utterances despite the rich higher level semantic and syntactic constraints found in these utterances. This finding suggests that perceptual learning of voices and its effect on language comprehension is a general phenomenon that operates in a variety of listening situations with different kinds of linguistic material. Familiarity with talker-specific information not only aids speech perception when higher level, top-down strategies are limited, but also when several sources of linguistic information are available to the listener. These findings suggest that the use of talker-specific information is important in general in the perception and comprehension of spoken language and is used in conjunction with other sources of information to derive a linguistic interpretation of a talker's utterance.

\section{GENERAL DISCUSSION}

The results of the present series of experiments demonstrate that perceptual learning of voices facilitates the analysis of the linguistic content of the signal. Listeners who learned to attend to talker-specific attributes of the speech signal were able to use that information to aid in the recovery of the linguistic content in the acoustic speech signal. This finding suggests at the broadest level that the perception of indexical or personal properties in the speech signal and the perception of linguistic properties are not independent, but rather are fundamentally linked in the perception of spoken language. Thus, acquiring sensitivity along the dimension of talker identity also increases perceptual sensitivity for other linguistic dimensions, suggesting that these dimensions are integral with respect to their perceptual underpinnings (Mullennix \& Pisoni, 1990). This demonstration of the influence of 
perceptual learning of talker identity on linguistic processing has implications not only for current theories of speech perception and spoken language processing, but also more generally for theories of perceptual learning and perception.

More specifically, the present series of experiments demonstrates that attention during perceptual learning must be specific to the perceptual task required of the listener. When confronted with an intelligibility task using isolated words, listeners who had attended during training to word level talker-specific attributes showed perceptual facilitation in recognition of isolated words. Listeners who had attended during training to sentencelevel talker-specific information showed little benefit on a word identification task, but displayed large familiar voice benefits in a sentence transcription task. These taskspecific aspects of the current investigation suggest that the transfer of perceptual learning of voice to linguistic processing requires that listeners learn about distinctiveness along just the talker-specific dimensions that will be relevant later. The implication of this finding is that different kinds of talker-specific information are available in different kinds of utterances and that all levels of talker-specific information are susceptible to the effects of perceptual learning.

The proposal that learning talker information can affect linguistic processing, while intuitive, is not presently addressed explicitly by any of the contemporary theories of speech perception and spoken language processing (Fowler, 1986; Liberman \& Mattingly, 1985; McClelland \& Elman, 1986; Nygaard \& Pisoni, 1995; Stevens \& Blumstein, 1978). Either explicitly or implicitly, theories of speech perception have traditionally dismissed talkers' voice in speech perception as a source of noise that must be discarded or separated from linguistic content. To the extent that talker-specific aspects of the signal have been studied, adjustments to variability introduced by talker-specific attributes of the signal have been characterized by the use of normalization procedures in which listeners make short-term automatic compensations for talker variability (Ladefoged \& Broadbent, 1957; Miller, 1989; Nearey, 1989). Our finding that learning a talker's voice makes their speech more intelligible suggests a very different interpretation of the role of talker variability in speech perception. The fact that attention to talker identity increases sensitivity to phonetic information in the signal suggests that both sources of information, indexical and linguistic, involve at least some of the same underlying attributes (Remez et al., 1997).

Beyond calling into question traditional assumptions about the role of talker identity in speech perception, the present set of findings suggest several conclusions about the nature of representation and processing of spoken language. First, our findings confirm that talker-specific information is retained along with linguistic information in long-term memory for linguistic events (Church \& Schacter, 1994; Goldinger, 1992; Nygaard et al., 1994; Palmeri et al., 1993; Pisoni, 1997). Detailed representa- tions of linguistic events appear to be retained in longterm memory, and linguistic categories may consist of collections of instance-specific exemplars (Goldinger, 1992, 1996; Hintzman, 1986; Nosofsky, 1987) rather than some type of abstract prototypical summary representation in which aspects of spoken language such as talker's voice (and speaking rate, vocal effort, etc., for that matter) are eliminated. However, our findings take this notion one step further. In addition to showing that talker information is retained in memory, these experiments also demonstrate that linguistic processing and the perception of talker identity are linked in a contingent fashion (Nygaard et al., 1994). Not only is talker information retained along with lexical information, but these two dimensions do not appear to be separable or independent in perception and attention (Mullennix \& Pisoni, 1990). There are important processing consequences for a shared or detailed representation of linguistic events. One of these consequences is that perceptual learning of voice identity can result in talker-specific sensitivity to linguistic content. Another consequence is that shared, detailed representations take linguistic representations out of the domain of abstract, symbolic units and into the domain of representation and memory for natural events and specific instances of these events (Brooks, 1978; Goldinger, 1992; Jacoby \& Brooks, 1984).

Second, the retention of detailed talker-specific information and its effect on linguistic processing has ramifications for the type of processing architecture and perceptual operations that must underlie speech and language perception. One of the most influential ideas in the area of language and cognitive architecture has centered on the notion of modularity (Fodor, 1983). Modules are specialpurpose, automatic, serial, cognitively impenetrable structures that process perceptual input quickly and reflexively (Garfield, 1987). As applied to language processing, a modular account of speech perception and word recognition assumes that language is processed by a special-purpose device that is concerned only with the linguistic aspects of spoken language. Higher level pragmatic or semantic knowledge are assumed to be outside the domain of the language module.

As applied to speech perception in particular, Liberman and Mattingly (1985) have argued for a "phonetic module" that operates exclusively on the linguistic aspects of the signal, quickly discarding acoustic information associated with nonlinguistic aspects. According to this view, the phonetic module should be impervious to the perceptual learning of talker identity. The perception of a talker's voice is assumed to have separate underlying representations and analyses from the perception of linguistic content. Given the present findings, however, it appears that the phonetic module does "know" something about the talker's voice.

One way to reconcile a modular account of language processing with the present findings is to assume that it is the perceptual normalization process or the set of "perceptual operations" which discard talker variability that 
is learned in our task. That is, talker-specific perceptual operations are retained or developed during the course of training, and listeners find speech from familiar talkers to be more intelligible than speech from unfamiliar talkers because they are better able to disentangle talker from linguistic information (Kolers, 1976; Kolers \& Ostry, 1974). The perceptual operations that are specifically associated with unraveling the variations introduced by particular talkers could be modified to become more efficient.

This account also preserves the distinction between voice recognition and linguistic processing. Evidence that this interpretation may be appropriate comes from studies of voice recognition in brain-damaged individuals (Van Lancker, 1991; Van Lancker et al., 1988). In a series of studies, Van Lancker and her colleagues have found that perception of the personal characteristics of speech appear to be subserved by the right hemisphere, whereas linguistic processing appears to be localized in the left hemisphere. This anatomical separation predicts a functional dissociation which our data appear to contradict by demonstrating an effect of talker familiarity on linguistic processing. However, if learning voices results in a modification of perceptual compensation operations, then hemispheric differences in identifying a talker's voice and linguistic content could be preserved while at the same time perceptual learning of voice would be shown to have an impact on linguistic processing. Thus, if learning involves facilitation of the unraveling of linguistic and voice information rather than some type of combined, detailed representational system - for example, of indexical and linguistic properties-then the distinctions between the two tasks could be preserved. If this account is correct, what listeners are learning during our perceptual learning task is a fine tuning of normalization procedures.

An alternative to this view would assume that the extraction of talker information and the extraction of linguistic information constitute a single perceptual ability that is no different from the extraction of surface and object characteristics in other modalities (Fowler, 1986; Nygaard \& Kalish, 1994). The reason that familiarity with a talker's voice affects linguistic processing is then a result of a common underlying code for perception and common perceptual operations for the perception of voice and the perception of phonetic content of the signal. Thus, during perceptual learning of talker's voice, listeners become highly skilled and attuned in recovering the consequences of dynamic vocal tract events. Any perceptual learning that increases distinctiveness or sensitivity to the dimension of talker identity would therefore increase sensitivity to linguistic aspects of the signal as well.

In summary, our findings demonstrate that perceptual learning of a taiker's voice influences the intelligibility of isolated words and words in sentences. Familiar voices are more intelligible than unfamiliar voices, and this dif- ference suggests that the dimensions along which talker identity varies are integrally related to the dimensions that subserve linguistic processing. Our findings showing a link in perceptual processing between the indexical and linguistic properties of speech constitute one of the first demonstrations of the important role that perceptual learning of talker information plays in the perception of spoken language.

\section{REFERENCES}

ABERCROMBIE, D. (1967). Elements of general phonetics. Chicago: Aldine.

assmanN, P. F., Nearey, T. M., \& Hogan, J. T. (1982). Vowel identification: Orthographic, perceptual, and acoustic aspects. Journal of the Acoustical Society of America, 71, 975-989.

Bradlow, A. R., NygaARd, L. C., \& Pisoni, D. B. (1995). On the contribution of in.tance-specific characteristics to speech perception. In C. Sorin, J. Mariani, H. Meloni, \& J. Schoentagen (Eds.), Levels in speech communication: Relations and interactions (pp. 13-24). Amsterdam: Elsevier.

Bradlow, A. R., Torretta, G. M., \& Pisoni, D. B. (1996). Intelligibility of normal speech I: Global and fine-grained acoustic-phonetic talker characteristics. Speech Communication, 20, 255-272.

Bricker, P. D., \& PruZansky, S. (1976). Speaker recognition. In N. J. Lass (Ed.), Contemporary issues in experimental phonetics (pp. 295326). New York: Academic Press.

BROOKS, L. (1978). Nonanalytic concept formation and memory for instances. In E. Rosch \& B. Lloyd (Eds.), Cognition and categorization (pp. 169-211). Hillsdale, NJ: Erlbaum.

Church, B. A., \& Schacter, D. L. (1994). Perceptual specificity of auditory priming: Implicit memory for voice intonation and fundamental frequency. Journal of Experimental Psychology: Learning, Memory, \& Cognition, 20, 521-533.

Cole, R. A., Coltheart, M., \& Allard, F. (1974). Memory of a speaker's voice: Reaction time to same- or different-voiced letters. Quarterly Journal of Experimental Psychology, 26, 1-7.

Costanzo, F. S., Markel, N. N., \& Costanzo, P. R. (1989). Voice quality profile and perceived emotion. Journal of Counseling Psychology, 16, 267-270.

Craik, F. I. M., \& KirSnER, K. (1974). The effect of speaker's voice on word recognition. Quarterly Journal of Experimental Psychology, 26, 274-284.

Creelman, C. D. (1957). The case of the unknown talker. Journal of the Acoustical Society of America, 29, 655.

DodDINGTON, G. R. (1985). Speaker recognition: Identifying people by their voices. Proceedings of the IEEE, 73, 1651-1664.

DUPOUX, E., \& GREEN, K. (1997). Perceptual adjustment to highly compressed speech: Effects of talker and rate changes, Journal of Experimental Psychology: Human Perception \& Performance, 23, 914-927.

EGAN, J. P. (1948). Articulation testing methods. Laryngoscope, 58 , 955-991.

FANT, G. (1973). Speech sounds and features. Cambridge, MA: MIT Press.

FODOR, J. A. (1983). The modularity of mind. Cambridge, MA: MIT Press.

FowLER, C. A. (1986), An event approach to the study of speech perception from a direct-realist perspective. Journal of Phonetics, 14, 3-28.

GARFIELD. J. L. (1987). Introduction: Carving the mind at its joints. In J. L. Garfield (Ed.), Modularity in knowledge representation and natural-language understanding (pp. 17-23). Cambridge, MA: MIT Press.

GARNER, W. (1974). The processing of information and structure. Hillsdale, NJ: Erlbaum.

Garvin, P. L., \& Ladefoged, P. L. (1963). Speaker identification and message identification in speech recognition. Phonetica, 9, 193-199. 
GEISELMAN, R. E. (1979). Inhibition of the automatic storage of speaker's voice. Memory \& Cognition, 7, 201-204.

Geiselman, R. E., \& Bellezza, F. S. (1976). Long-term memory for speaker's voice and source location. Memory \& Cognition, 4, 483-489.

Geiselman, R. E., \& BellezZA, F. S. (1977). Incidental retention of speaker's voice. Memory \& Cognition, 5, 658-665.

Geiselman, R. E., \& Crawley, J. M. (1983). Incidental processing of speaker characteristics: Voice as connotative information. Journal of Verbal Learning \& Verbal Behavior, 22, 15-23.

GiBson, E. J. (1969). Principles of perceptual learning and development. New York: Appleton-Century-Crofts.

GiBson, E. J. (1991). An odyssey in learning and perception. Cambridge, MA: MIT Press.

GiBson, J. J., \& GiBson, E. J. (1955). Perceptual learning: Differentiation or enrichment? Psychological Review, 62, 32-41.

GOLDINGER, S. D. (1992). Words and voices: Implicit and explicit memory for spoken words (Research on Speech Perception Tech. Rep. No. 7). Bloomington: Indiana University, Department of Psychology.

GoldiNGER, S. D. (1996). Words and voices: Episodic traces in spoken word identification and recognition memory. Journal of Experimental Psychology: Learning, Memory, \& Cognition, 22, 1166-1183.

Goldinger, S. D., PISONI, D. B., \& LogAN, D. B. (1991). The nature of talker variability effects on recall of spoken word lists. Journal of Experimental Psychology: Learning, Memory, \& Cognition, 17, $152-162$.

GOLDSTONE, R. (1994). Influences of categorization on perceptual discrimination. Journal of Experimental Psychology: General, 123, 178-200.

Greenspan, S. L., Nusbaum, H. C., \& Pisoni, D. B. (1988). Perceptual learning of synthetic speech produced by rule. Journal of Experimental Psychology: Learning, Memory, \& Cognition, 14, 421-433.

HALL, G. (1991). Perceptual and associative learning. Oxford: Oxford University Press, Clarendon Press.

HALLE, M. (1985). Speculations about the representation of words in memory. In V. A. Fromkin (Ed.), Phonetic linguistics (pp. 101-114). New York: Academic Press.

HinTZMAN, D. L. (1986). "Schema abstraction" in a multiple trace memory model. Psychological Review, 93, 411-428.

House, A. S., Williams, C. E., Hecker, M. H. L., \& Kryter, K. D. (1965). Articulation-testing methods: Consonantal differentiation with a closed-response set. Journal of the Acoustical Society of America, 37, 158-166.

InSTITUTE OF ELECTRICAL AND ELECTRONICS ENGINEERS (1969). IEEE recommended practice for speech quality measurements (IEEE Report No. 297). New York: Author.

JACOBY, L. L., \& BROOKS, L. R. (1984). Nonanalytic cognition: Memory, perception, and concept learning. In G. H. Bower (Ed.), The psychology of learning and motivation (Vol. 18, pp. 1-47). New York: Academic Press.

JoHnson, K. (1990). The role of perceived speaker identity in $F 0$ normalization of vowels. Journal of the Acoustical Society of America, 88, 642-654.

Joos, M. A. (1948). Acoustic phonetics. Language, 24(Suppl. 2), 1-136.

Kolers, P. A. (1976). Pattern analyzing memory. Science, 191, 12801281.

KolERS, P. A., \& Ostry, D. J. (1974). Time course of loss of information regarding pattern analyzing operations. Journal of Verbal Learning \& Verbal Behavior, 13, 599-612.

KUHL, P. K. (1991). Human adults and human infants show a "perceptual magnet effect" for the prototypes of speech categories, monkeys do not. Perception \& Psychophysics, 50, 93-107.

KUHL, P. K. (1992). Psychoacoustics and speech perception: Internal standards, perceptual anchors, and prototypes. In L. A. Werner \& E. W. Rubel (Eds.), Developmental psychoacoustics (pp. 293-332). Washington, DC: APA Press.

LABOv, W. (1972). Sociolinguistic patterns. Philadelphia: University of Pennsylvania Press.

LADEFOGED, P. (1980). What are linguistic sounds made of? Language, 56, 485-502.

LADEFOGED, P., \& BROADBENT, D. E. (1957). Information conveyed by vowels. Journal of the Acoustical Society of America, 29, 98-104.

LAVER, J. (1989). Cognitive science and speech: A framework for re- search. In H. Schnelle \& N. O. Bernsen (Eds.), Logic and linguistics: Research directions in cognitive science. European perspectives (Vol. 2, pp. 37-70). Hillsdale, NJ: Erlbaum.

Laver, J., \& Trudgill, P. (1979). Phonetic and linguistic markers in speech. In K. R. Scherer \& H. Giles (Eds.), Social markers in speech (pp. 1-32). Cambridge: Cambridge University Press.

LAWRENCE, D. H. (1949). Acquired distinctiveness of cues: I. Transfer between discriminations on the basis of familiarity with the stimulus. Journal of Experimental Psychology, 39, 770-784.

Legge, G. E., Grossmann, C., \& Pieper, C. M. (1984). Learning unfamiliar voices. Journal of Experimental Psychology: Learning, Memory, \& Cognition, 10, 1-36.

Liberman, A. M., \& MatTingly, I. G. (1985). The motor theory of speech perception revised. Cognition, 21, 1-36.

LIGHTFOOT, N. (1989). Effects of talker familiarity on serial recall of spoken word lists (Research on Speech Perception Progress Report No. 15). Bloomington: Indiana University, Department of Psychology.

Lively, S. E., LogaN, J. S., \& Pisoni, D. B. (1993). Training Japanese listeners to identify English / $/$ and $/ 1 /:$ II. The role of phonetic environment and talker variability in learning new perceptual categories. Journal of the Acoustical Society of America, 94, 1242-1255.

Lively, S. E., Pisoni, D. B., Yamada, R. A., Tohkura, Y., \& YAMADA, T. (1994). Training Japanese listeners to identify English $/ \mathbf{r} /$ and /1/: III. Long-term retention of new phonetic categories. Journal of the Acoustical Society of America, 96, 2076-2087.

Logan, J. S., Lively, S. E., \& Pisoni, D. B. (1991). Training Japanese listeners to identify English / $\mathrm{r} /$ and /1/: A first report. Journal of the Acoustical Society of America, 89, 874-886.

LuCE, P. A., Pisoni, D. B., \& Goldinger, S. D. (1990). Similarity neighborhoods of spoken words. In G. T. M. Altmann (Ed.), Cognitive models of speech processing: Psycholinguistic and computational perspectives (pp. 122-147). Cambridge, MA: MIT Press.

Markel, N. N., Bein, M. F., \& Phillis, J. (1973). The relationship between words and tone-of-voice. Language \& Speech, 16, 15-21.

Martin, C. S., Mullennix, J. W., Pisoni, D. B., \& Summers, W. V. (1989). Effects of talker variability on recall of spoken word lists. Journal of Experimental Psychology: Learning, Memory, \& Cognition, 15 , 676-681.

McClelland, J. L., \& Elman, J. L. (1986). The TRACE model of speech perception. Cognitive Psychology, 18, 1-86.

MiLLER, J. D. (1989). Auditory-perceptual interpretation of the vowel. Journal of the Acoustical Society of America, 85, 2114-2134.

Mullennix, J. W., \& Pisoni, D. B. (1990). Stimulus variability and processing dependencies in speech perception. Perception \& Psychophysics, 47, 379-390.

Mullennix, J. W., Pisoni, D. B., \& Martin, C. S. (1989). Some effects of talker variability on spoken word recognition. Journal of the Acoustical Society of America, 85, 365-378.

MURRAY, I. R., \& ARNOTT, J. L. (1993). Toward the simulation of emotion in synthetic speech: A review of the literature on human vocal emotion. Journal of the Acoustical Society of America, 93, 1097-1108.

NEAREY, T. M. (1989). Static, dynamic, and relational properties in vowel perception. Journal of the Acoustical Society of America, $\mathbf{8 5}$, 2088-2113.

Nosofsky, R. M. (1987). Attention and learning processes in the identification and categorization of integral stimuli. Journal of Experimental Psychology: Learning, Memory, \& Cognition, 15, 700-708.

Nusbaum, H. C., Pisoni, D. B., \& Davis, D. K. (1984). Sizing up the Hoosier mental lexicon: Measuring the familiarity of 20,000 words (Research on Speech Perception Progress Report No. 10). Bloomington: Indiana University, Department of Psychology.

NyGaARD, L. C., \& KaLiSh, M. L. (1994). Modeling the effect of learning voices on the perception of speech. Journal of the Acoustical Society of America, 95, 2873.

NygaARD, L. C., \& Pisoni, D. B. (1995). Speech perception: New directions in research and theory. In J. L. Miller \& P. D. Eimas (Eds.), Handbook of perception and cognition: Vol. II. Speech, language and communication (pp. 63-96). New York: Academic Press.

Nygaard, L. C., Sommers, M. S., \& Pisoni, D. B. (1994). Speech perception as a talker-contingent process. Psychological Science, 5, 42-46. Nygaard, L. C., Sommers, M. S., \& Pisoni, D. B. (1995). Effects of 
stimulus variability on perception and representation of spoken words in memory. Perception \& Psychophysics, 57, 989-1001.

Palmeri, T. J., Goldinger, S. D., \& Pisoni, D. B. (1993). Episodic encoding of voice attributes and recognition memory for spoken words. Journal of Experimental Psychology: Learning, Memory, \& Cognition, 19, 309-328.

PETERS, R. W. (1955a). The effect of length of exposure to speaker's voice upon listener reception. In Joint Project Report No. 44 (pp. 1-8). Pensacola, FL: U.S. Naval School of Aviation Medicine.

PETERS, R. W. (1955b). The relative intelligibility of single-voice and multiple-voice messages under various conditions of noise. In Joint Project Report No. 56 (pp. 1-9). Pensacola, FL: U.S. Naval School of Aviation Medicine.

Peterson, G. E., \& Barney, H. L. (1952). Control methods used in a study of the vowels. Journal of the Acoustical Society of America, 24, 175-184.

Pisoni, D. B. (1993). Long-term memory in speech perception: Some new findings on talker variability, speaking rate, and perceptual learning. Speech Communication, 13, 109-125.

PISONI, D. B. (1997). Some thoughts on "normalization" in speech perception. In K. Johnson \& J. W. Mullennix (Eds.), Talker variability in speech processing (pp. 9-32). San Diego: Academic Press.

Pollack, I., Pickett, J. M., \& Sumby, W. H. (1954). On the identification of speakers by voice. Journal of the Acoustical Society of America, 26, 403-406.

Remez, R. E., Fellowes, J. M., \& Rubin, P. E. (1997). Talker identification based on phonetic information. Journal of Experimental Psychology: Human Perception \& Performance, 23, 651-666.

SCHACTER, D. L. (1990). Perceptual representation systems and implicit memory: Toward a resolution of the multiple memory systems debate. In A. Diamond (Ed.), Development and neural bases of higher cortical functions (Annals of the New York Academy of Sciences, Vol. 608, pp. 543-571). New York: New York Academy of Sciences.

Schwab, E. C., Nusbaum, H. C., \& Pisoni, D. B. (1985). Some effects of training on the perception of synthetic speech. Human Factors, 27, $395-408$.

Shankweiler, D. P., STrange, W., \& Verbrugge, R. R. (1977). Speech and the problem of perceptual constancy. In R. Shaw \& J. Bransford (Eds.), Perceiving, acting, and knowing: Toward an ecological psychology (pp. 315-345). Hillsdale, NJ: Erlbaum.

ShePARD, R. N., \& TEGHTSOONIAN, M. (1961). Retention of information under conditions approaching a steady state. Journal of Experimental Psychology, 62, 302-309.

Sommers, M. S., NygaARD, L. C., \& Pisoni, D. B. (1994). Stimulus variability and spoken word recognition: I. Effects of variability in speaking rate and overall amplitude. Journal of the Acoustical Society of America, 96, 1314-1324.

STEvens, K. N., \& BLUMSTEIN, S. E. (1978). Invariant cues for place of articulation in stop consonants. Journal of the Acoustical Society of America, 64, 1358-1368.

Strange, W., \& DitTmanN, S. (1984). Effects of discrimination training on the perception of $/ \mathrm{r}-1 /$ by Japanese adults learning English. Perception \& Psychophysics, 36, 131-145.

SUMMERFIELD, Q. (1975). Acoustic and phonetic components of the influence of voice changes and identification times for CVC syllables. In Report on research in progress in speech perception (Vol. 2, pp. 73-
98). Belfast, Northern Ireland: The Queen's University of Belfast, Department of Psychology.

Summerfield, Q., \& HAGGARD, M. P. (1973). Vocal tract normalization as demonstrated by reaction times. In Report of speech research in progress (Vol. 2, pp. 12-23). Belfast, Northern Ireland: The Queen's University of Belfast.

Thompson, C. P. (1985). Voice identification: Speaker identifiability and a correction of the record regarding sex effects. Human Learning: Journal of Practical Research \& Applications, 4, 19-27.

VAN LANCKER, D. (1991). Personal relevance and the human right hemisphere. Brain \& Cognition, 17, 64-92.

Van Lancker, D., Cummings, J. L., Kreiman, J., \& Dobkin, B. H. (1988). Phonagnosia: A dissociation between familiar and unfamiliar voices. Cortex, 24, 195-209.

VAN LANCKER, D., \& KREIMAN, J. (1987). Voice discrimination and recognition are separate abilities. Neuropsychologia, 25, 829-854.

VAN LANCKer, D., KREIMAN, J., \& EMmOREY, K. (1985). Familiar voice recognition: Patterns and parameters: Part I. Recognition of backward voices. Journal of Phonetics, 13, 19-38.

VAN LANCKer, D., Kreiman, J., \& Wickens, T. (1985). Familiar voice recognition: Patterns and parameters. Part II. Recognition of ratealtered voices. Journal of Phonetics, 13, 39-52.

Verbrugge, R. R., Strange, W., Shankweiler, D. P., \& Edman, T. R. (1976). What information enables a listener to map a talker's vowel space? Journal of the Acoustical Society of America, 60, 198-212.

WEENINK, D. J. M. (1986). The identification of vowel stimuli from men, women, and children. Proceedings from the Institute of Phonetic Sciences of the University of Amsterdam, 10, 41-54.

Williams, C. E. (1964). The effects of selected factors on the aural identification of speakers. In Report EDS-TDR-65-153 (Section III). Hanscom Field, MA: Air Force Systems Command, Electronic Systems Division.

WOHLWLL, J. F. (1958). The definition and analysis of perceptual learning. Psychological Review, 65, 283-295.

\section{NOTES}

1. We chose to treat perceptual learning as a categorical variable here because we failed to find an orderly relationship between amount of perceptual learning of voice and absolute word intelligibility. For example, although female speakers were more intelligible overall than male speakers, male speakers were more identifiable than female speakers. Thus, differences in baseline intelligibility among speakers as well as in baseline identifiability make the relationship between learning and intelligibility complex. To test this assumption, regression analyses were performed. It was determined that treating learning as a continuous variable violated the assumptions of the analysis. For consistency, perceptual learning is treated as a categorical variable in Experiments 2 and 3 as well.

2. One listener from the control group fell just short of $70 \%$ correct on the 9 th day of training. However, his/her performance rose to $75 \%$ correct on the generalization test and consequently, this listener was included with the "good" learners from the control group

(Manuscript received October 14, 1996; revision accepted for publication May 4, 1997.) 